\title{
Effects of an Asymmetric Friction on the Nonlinear Equilibration of a Baroclinic System
}

\author{
Pascal Rivière and Patrice Klein \\ Laboratoire de Physique des Océans, Institut Français de Recherche pour l'Exploitation de la Mer, Plouzane, France
}

(Manuscript received 29 February 1996, in final form 12 November 1996)

\begin{abstract}
Following some recent linear and nonlinear studies the authors examine, using numerical simulations of a classical two-layer model, the effect of an asymmetric friction on the nonlinear equilibrium of moderately unstable baroclinic systems. The results show that the presence of an asymmetric friction leads to a significant wave scale selection: "long" waves (in terms of their zonal wavelengths) emerge with a traditional asymmetric friction (with the upper layer less viscous than the lower layer), while only "short" waves dominate with a nontraditional asymmetric friction (with the lower layer less viscous than the upper layer). The role of the nonlinear interactions and, more precisely, the effects of an asymmetric friction on the wave-mean flow and wave-wave interactions and their consequences on the wave scale selection are examined.
\end{abstract}

\section{Introduction}

Ekman friction usually provides a dissipative sink of energy and potential vorticity of baroclinic waves. However, in some cases and, in particular, in the presence of a viscous vertical asymmetry, friction can act as a source of energy of baroclinic waves. Indeed, in those cases asymmetric friction produces small alterations in the structure of the waves and, in particular, an additional secondary circulation that can destabilize an otherwise stable baroclinic flow regime. This mechanism, first studied by Holopainen (1961) and interpreted as a "frictional instability," was carefully examined by Pedlosky (1983) and other authors.

Their results revealed that the asymmetric friction effect is efficient mostly for the weakly unstable systems. This specific efficiency is, however, especially important for most geophysical baroclinic systems because, in the process of nonlinear equilibration of baroclinic waves, the statistically equilibrated zonal flow is usually found to be weakly supercritical according to the linear theory. An illustration of the efficiency of these effects on geostrophic turbulent systems is given by the study of Hua and Haidvogel (1986) in which their energy budget analysis well displays the dissipation terms of the baroclinic modes acting as an energy source for these modes (see their Fig. 11). The relevance of this destabilizing effect of asymmetric friction for geophysical systems, in addition to the uncertainty about

Corresponding author address: Dr. Patrice Klein, LPO, IFREMER, B.P. 70, 29280 Plouzane, France.

E-mail: pklein@ifremer.fr the Ekman friction magnitude and its degree of asymmetry (Palmer et al. 1986; McFarlane 1987), has been the motivation of the large number of linear and nonlinear studies devoted to this topic.

The linear studies have shown that the presence of an asymmetric friction can lead to a wave scale selection: a traditional asymmetric friction (with the upper layer less viscous than the lower layer) exerts an additional destabilizing effect on short waves and a stabilizing effect on long waves. The opposite is true for a nontraditional asymmetric friction (with the upper layer more viscous than the lower layer). Is this wave scale selection damped or amplified by the nonlinearities? The nonlinear studies have not been able to address this question since they have considered baroclinic systems with only a narrow spectral waveband, including up to three unstable short waves (Klein 1990). However, they have shown that an asymmetric friction significantly affects the nonlinear equilibration of weakly or moderately unstable systems. Consequently, in this paper we reexamine the effects of an asymmetric friction on the nonlinear equilibration of baroclinic systems with a particular focus on the wave scale selection. For that purpose, the baroclinic systems considered involve a large spectral wave band including both long and short waves.

A short review of the main results of the linear and nonlinear studies is given in the next section. Section 3 describes the model used and the parameter setting chosen in this study. The main results are discussed in section 4 and their robustness is examined in section 5. Section 6 investigates some mechanisms involved in the wave scale selection revealed by the nonlinear results. 


\section{A short review}

\section{a. Linear studies}

Most of the studies devoted to the frictional destabilization of baroclinic shear use the generic Phillips (1954) model. It is a two-layer quasigeostrophic model with a channel geometry on the $\beta$ plane whose variables and parameters are nondimensionalized by a scaling velocity $V$ and the width of the channel $L$ (see Pedlosky 1987 for details). Let us define $F$ as the Froude number,
$U_{s}=\left(U_{1}-U_{2}\right) / 2$ the uniform baroclinic shear introduced as a forcing (with $U_{1}$ and $U_{2}$ the velocity in the upper and lower layer), and $r_{1} / 2$ and $r_{2} / 2$ the dissipation rates produced by upper and lower Ekman layers. For the sake of simplicity, let us choose, by a Galilean transformation, to have $U_{1}=-U_{2}$. If $\phi_{B}=\left(\phi_{1}+\phi_{2}\right) / 2$ and $\phi_{T}=\left(\phi_{1}-\phi_{2}\right) / 2$ are the streamfunctions of, respectively, the barotropic and baroclinic components of the perturbated flow, the nondimensional governing potential vorticity equations can be written as

$$
\begin{array}{r}
\frac{\partial \Delta \phi_{B}}{\partial t}+J\left(\phi_{B}, \Delta \phi_{B}\right)+J\left(\phi_{T}, \Delta \phi_{T}\right)=-\beta \frac{\partial \phi_{B}}{\partial x}-U_{s} \frac{\partial \Delta \phi_{T}}{\partial x}-\frac{\left(r_{1}+r_{2}\right)}{4} \Delta \phi_{B}-\frac{\left(r_{1}-r_{2}\right)}{4} \Delta \phi_{T} \\
\frac{\partial\left(\Delta \phi_{T}-2 F \phi_{T}\right)}{\partial t}+J\left(\phi_{B}, \Delta \phi_{T}-2 F \phi_{T}\right)+J\left(\phi_{T}, \Delta \phi_{B}\right)=-\beta \frac{\partial \phi_{T}}{\partial x}-U_{s} \frac{\left(\partial \Delta \phi_{B}+2 F \phi_{B}\right)}{\partial x}-\frac{\left(r_{1}+r_{2}\right)}{4} \Delta \phi_{T}-\frac{\left(r_{1}-r_{2}\right)}{4} \Delta \phi_{B},(2)
\end{array}
$$

where $J$ is the Jacobian operator and $\Delta$ the Laplacian operator. The boundary conditions are

$$
\begin{aligned}
\phi_{B, T}(0, y, t) & =\phi_{B, T}(\gamma, y, t), \\
\frac{\partial \phi_{B, T}}{\partial x} & =0, \quad \frac{\partial}{\partial t} \int_{0}^{\gamma} \frac{\partial \phi_{B, T}}{\partial y} d x=0 \quad \text { at } y=0,1,
\end{aligned}
$$

with $\gamma$ the length of the channel nondimensionalized by its width. Holopainen (1961), using a linearized version of this model with both a $\beta$ effect and an asymmetric friction, first showed how a weak friction can destabilize an otherwise subcritical baroclinic shear flow. The subsequent studies revealed that what he called a "frictional instability" actually involves two distinct destabilizing processes. Indeed, Romea (1977) and Pedlosky (1981) showed that a weak symmetric friction, when associated with a $\beta$ effect, is capable by itself of destabilizing the long waves otherwise stabilized in the inviscid limit. The additional destabilization provided by the specific asymmetric character of the friction has been revealed by later studies as Pedlosky (1983), Wang (1990), and Weng and Barcilon (1991). This specific effect of an asymmetric friction can be illustrated by considering a very simple situation with no $\beta$ effect in the weak viscosity limit and at the marginal stability threshold for the inviscid case (i.e., $2 F=a^{2}$ with $a$ the total wavenumber). In that situation the only linear terms to be considered in Eqs. (1) and (2) are the ones underbraced. Then a linear stability of the resulting equations, when $r_{1}+r_{2} \approx\left|r_{1}-r_{2}\right| \ll U_{s} k$ and using $\phi=A e^{i k(x-c t)}$ with $c=c_{r}+i c_{i}$, leads to

$$
\begin{aligned}
c_{r} & \approx \frac{1}{4} \sqrt{\frac{\left|r_{1}-r_{2}\right| U_{s}}{k}} \\
c_{i} & \approx-\frac{3}{16} \frac{r_{1}+r_{2}}{k}+\frac{1}{4} \sqrt{\frac{\left|r_{1}-r_{2}\right| U_{s}}{k} .}
\end{aligned}
$$

Thus in the weak viscosity limit, when the dissipation timescale is much larger than the advective timescale, the part of $k c_{i}$ associated with the frictional destabilization is larger than the damping rate due to the dissipative effects. A physical explanation is that the frictional asymmetry produces a vertical phase shift of the wave large enough for this wave to be capable of releasing the available potential energy of the baroclinic shear flow at a rate larger than the dissipation rate.

In a more general situation, the linear studies of Wang (1990) and Weng and Barcilon (1991) performed in the weak viscosity limit have revealed the specific effects of an asymmetric friction on the scale selection of the unstable waves. Let us summarize their results. Presence of an asymmetric friction leads to a destabilization of the short waves. This is found with or without the $\beta$ effect. Furthermore, with the $\beta$ effect and a westerly shear, a traditional asymmetric dissipation produces an additional destabilization of the short waves and tends to stabilize the long waves. The opposite is found for a nontraditional asymmetric dissipation with a westerly shear. Finally the effects of traditional and nontraditional asymmetric dissipation are interchanged for an easterly shear. This is due to a symmetry property displayed by the potential vorticity equations (1) and (2) in the $\beta$ plane that indicates that a nontraditional asymmetric friction associated with a westerly baroclinic shear is equivalent to a traditional asymmetric friction with an easterly baroclinic shear. Furthermore, as mentioned above, all the linear results emphasize that the effects of an asymmetric friction and the resulting wave scale selection are significant, mostly for the weakly unstable systems. Indeed for moderately or strongly unstable baroclinic systems, that is, when the supercriticality is $O(1)$, no such significant wave scale selection occurs since the large growth rates of the most unstable 

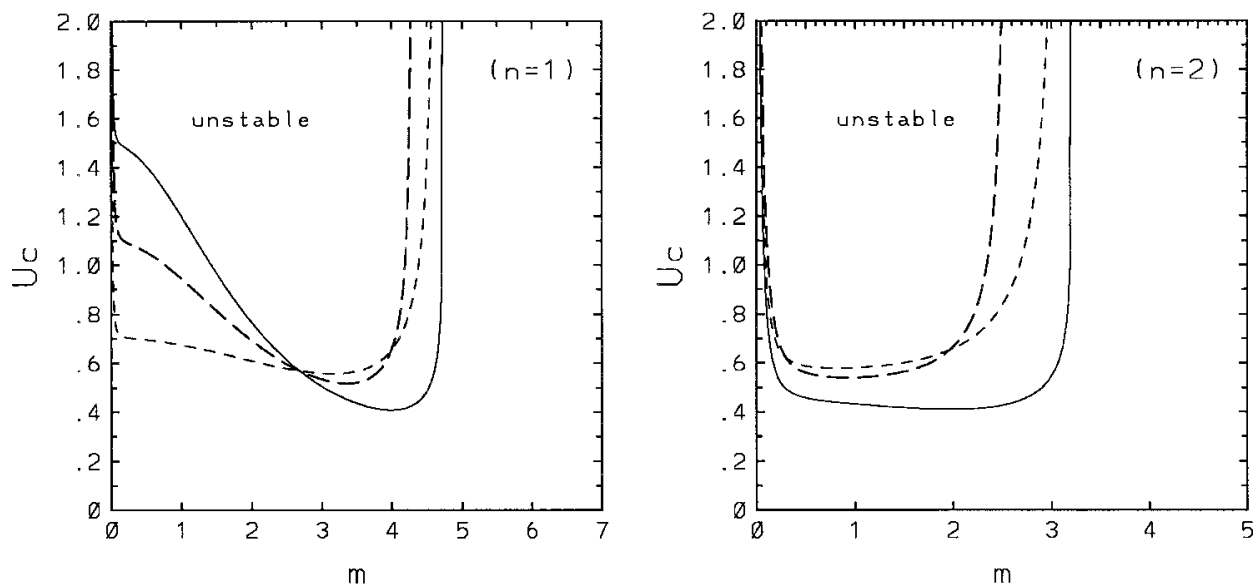

FIG. 1. Critical current shear $\left(U_{c}\right)$ as a function of the zonal wavenumber $(m)$ with (a) $n=1$ and (b) $n$ $=2$ when the spectral representation (4) is used. Long-dashed, solid, and short-dashed lines respectively refer to symmetric $\left(r_{1}=r_{2}\right)$, traditional $\left(r_{1}=0.2 r_{2}\right)$, and nontraditional $\left(r_{1}=5 r_{2}\right)$ asymmetric dissipation. Other parameters are $F=27.6, \beta=31.4$, and $\bar{r} / 2=\left(r_{1}+r_{2}\right) / 4=0.1$.

waves are almost unaffected by the presence of an asymmetric friction.

The important effects of an asymmetric friction are illustrated by Figs. 1 and 2 that show results from a linear stability analysis for baroclinic systems with characteristics close to the ones considered in this study. Figure 1 displays the critical shear required for baroclinic instability for three dissipative cases. It reveals the destabilizing effects on short waves (in terms of their total wavenumber) induced by an asymmetric friction: the short-wave cutoff in the presence of a viscous asymmetry has a larger value than in the symmetric case. Furthermore, the long waves are stabilized (destabilized) by a traditional (nontraditional) asymmetric friction. Lastly, the minimum critical shear is significantly reduced with a traditional asymmetry. Let us stress again that this is the combined effect of the frictional asym-

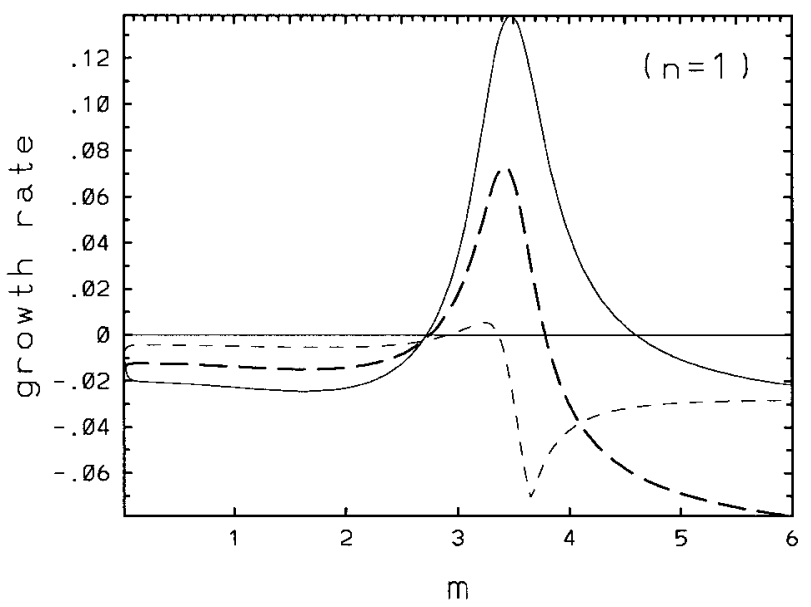

metry and $\beta$, which makes the baroclinic system to be affected by the sense of the asymmetry. Figure 2 reveals that for a weakly unstable system $\left(U_{s} \approx U_{c}\right)$ the scales and growth rates of the most unstable waves strongly depend on the presence and the sense of an asymmetric friction. Note that this wave scale selection for weakly unstable systems is increased by the discrete character of the waveband spectrum. On the other hand, for a moderately unstable system $\left(U_{s} \gg U_{c}\right)$, Fig. 3 clearly shows that the growth rates of the most unstable waves are unaffected by an asymmetric friction.

\section{b. Nonlinear studies}

The specific effects of an asymmetric friction on the nonlinear equilibration of baroclinic waves was first studied by Pedlosky (1983) in the weak viscosity limit.

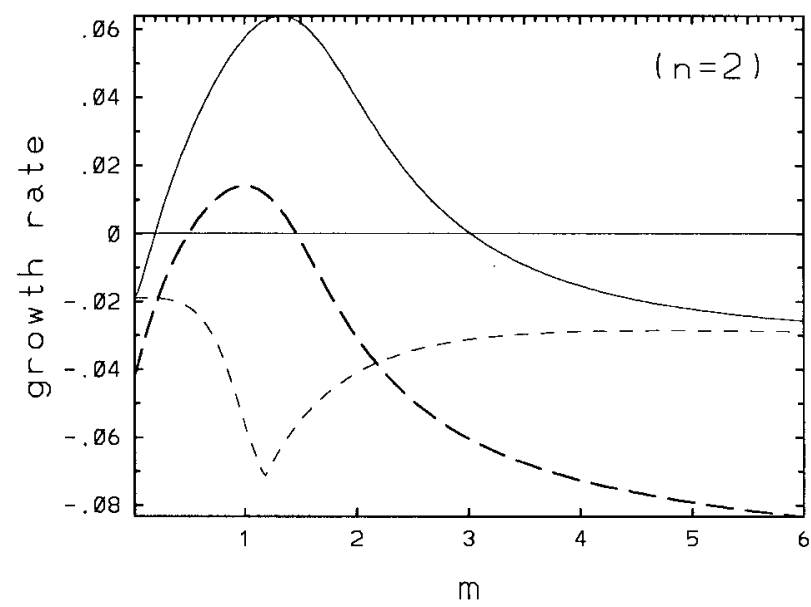

FIG. 2. Growth rate of the baroclinic waves as a function of their zonal wavenumber (m) with (a) $n=1$ and (b) $n=2$ when $U_{\mathrm{s}}=0.56$. Long-dashed, solid, and short-dashed lines respectively refer to symmetric $\left(r_{1}=r_{2}\right)$, traditional $\left(r_{1}=0.2 r_{2}\right)$, and nontraditional $\left(r_{1}=5 r_{2}\right)$ asymmetric dissipation. Other parameters are $F=27.6, \beta=31.4$, and $\bar{r} / 2=\left(r_{1}+r_{2}\right) / 4=0.1$. 

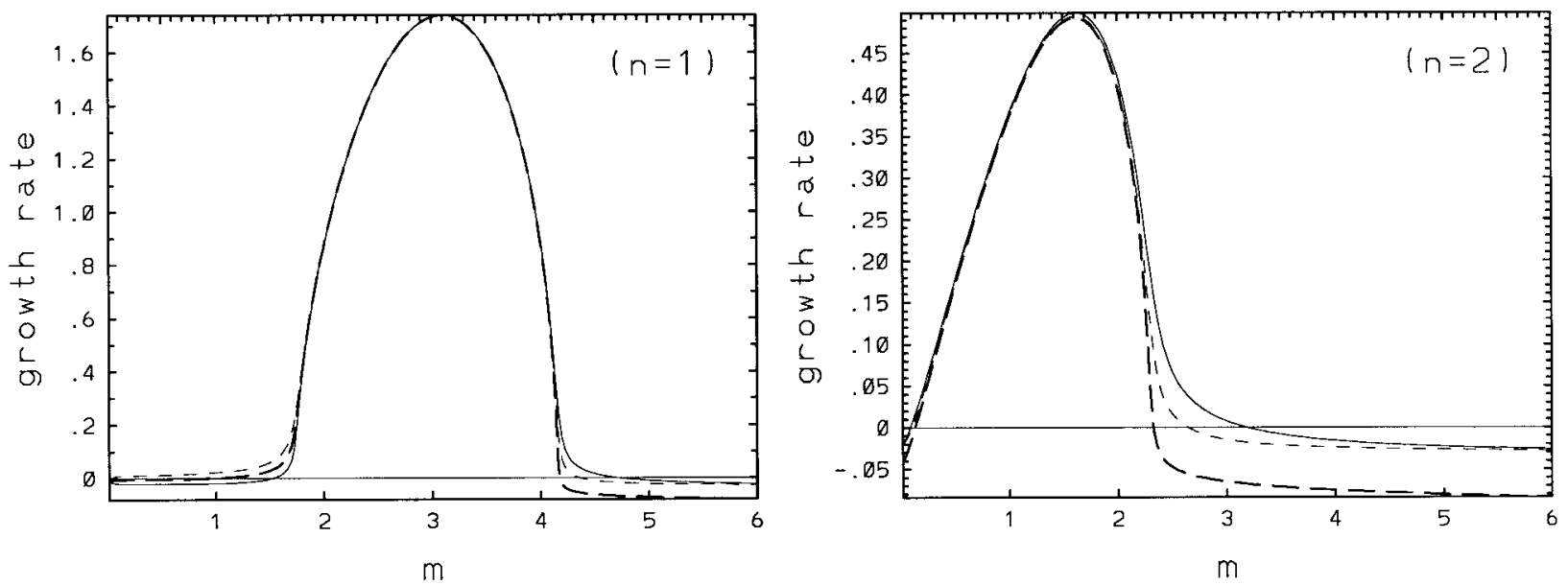

FIG. 3. As in Fig. 2 but when $U_{s}=0.95$.

He used the Phillips model on the $f$ plane to study the finite-amplitude dynamics of a weakly unstable baroclinic wave. In the absence of an Ekman dissipation in the upper layer, the nonlinear equilibration displays three distinct stages: First, the unstable baroclinic wave grows exponentially up to a vacillating finite amplitude state. Then, under the destabilizing influence of friction the amplitude of the vacillating wave slowly increases with time. During this intermediate stage there is a competition between the destabilizing influence of friction and the stabilizing effects of the nonlinearities. After a considerably longer time, the dissipative role of the friction becomes manifest. During this last stage the wave amplitude slowly diminishes and finally vanishes completely. The final state is wave free but the mean field displays a new zonal flow of lowered available potential energy. When considering the equation for the mean flow correction, Pedlosky argues that this result should hold even with $\beta$ since there is no dissipative sink for the potential vorticity in the upper layer.

The robustness of this result was checked and extended by Chou and Loesch (1986a) in the context of the Eady model in the $f$ plane with the upper boundary being stress free. They showed that Pedlosky's (1983) result is robust in a large region of the dissipationsupercriticality parameter space. In another paper, Chou and Loesch (1986b) explored the nonlinear equilibration in the presence of an asymmetric friction by varying the degree of asymmetry, $\alpha=\left(r_{1}-r_{2}\right) /\left(r_{1}+r_{2}\right)$. With a symmetric friction $(\alpha=0)$ the wave grows and then always reaches a steady equilibrated state. The nonlinear equilibration is quite different with the presence of a frictional asymmetry $(0<|\alpha|<1)$ : the first stage is very close to the one found with a free surface, displaying a single hump followed by a nearly wave-free state. However, during the nearly wave-free state the mean field gradually builds up because of the nonzero (although small) dissipation in the upper boundary, which then leads to an abrupt second growth of the wave. This leads to a final stage consisting of a chaotic or regular vacillating state. The amplitude of the chaotic vacillations are found to be all the more significant as the degree of asymmetry is high and the ratio of the mean dissipation to the supercriticality is small. These results reveal the strong competition between the destabilizing effects of friction and the nonlinear interactions that occurs even for $O(1)$ supercriticality. It is interesting to note that, although their system involved only one unstable baroclinic wave, Chou and Loesch found a significant sensitivity of their results to the spectral truncation when the asymmetry is high, which displays the importance of the wave-mean flow and wavewave interactions involving stable modes.

The nonlinear equilibration of three unstable baroclinic waves was studied by Mak (1987) using a twolayer $\beta$-plane model. His study confirms that, with a nonzero $\beta$ effect, not only the presence of a viscous asymmetry but also the sense of this asymmetry strongly influences the character of the nonlinear equilibration. With a traditional asymmetric friction the equilibrated state tends to be a steady multiple-wave field. With a nontraditional asymmetric friction the equilibrated state takes the form of a triad limit cycle that becomes unstable and leads to chaos as the degree of frictional asymmetry is increased. Only one traditional asymmetric case was examined. However, the large number of simulations performed in the symmetric and nontraditional asymmetric cases clearly displays the strong effect of an asymmetric friction on the nonlinear equilibration and, in particular, on the appearance of chaotic vacillations. This again reveals the competition between the destabilizing effects of the friction and the nonlinear interactions. However, as in the preceding studies, the spectral wave band considered (with only three unstable short waves) is small.

One interesting feature rationalized by Pedlosky (1983) and discussed by Mak (1987) is the existence of a barotropic zonal jet induced by the presence of unequal 
Ekman pumping at the horizontal boundaries. This barotropic jet can be understood by considering the steady form of the potential vorticity equation for the barotropic part of the mean zonal flow correction. Indeed, for weakly unstable systems involving only one wave, the nonlinear term related to the Reynolds flux is identically zero and this equation [deduced from (1)] in its steady form leads to

$$
\frac{\partial^{2} \Phi_{B}}{\partial y^{2}}=-\frac{r_{1}-r_{2}}{r_{1}+r_{2}} \frac{\partial^{2} \Phi_{T}}{\partial y^{2}}
$$

with $\Phi_{B}$ and $\Phi_{T}$ respectively the barotropic and baroclinic part of the mean flow correction. Since, when $U_{s}$ $>0$, the baroclinic part of the mean flow correction is westward, a traditional (nontraditional) asymmetric friction induces a westward (eastward) barotropic zonal jet. This result has been found by Mak (1987) to still hold in the $\beta$ plane for baroclinic systems with an $O(1)$ supercriticality involving several unstable waves, which means that the Reynolds stress flux induced by the wave-wave interactions is still very small for those systems whose basic state has no meridional shear.

No study has specifically examined the effects of an asymmetric friction on the nonlinear equilibrium of realistic atmospheric or oceanic flows. However, within the context of our study, it is worthwhile to mention some particular results found in Hua and Haidvogel (1986), Cai and Mak (1990b), Cehelsky and Tung (1991), and Whitaker and Barcilon (1995). Hua and Haidvogel (1986) examined some properties of the stratified quasigeostrophic turbulence using a three-dimensional spectral model with oceanic parameters and a stress-free upper boundary. Their energy budget clearly shows that the main energy source of the baroclinic modes of the dominant waves is the baroclinic instability generation term and the bottom friction term (with the same order of magnitude). These terms are balanced by the nonlinear terms. The barotropic mode is balanced between the nonlinear terms (acting as a source) and the bottom friction term. However, no partition of the nonlinear terms in wave-wave and wave-mean flow interactions has been done. Cai and Mak (1990b) using the Phillips model with a symmetric dissipation concluded that the dominant low-frequency atmospheric waves are maintained primarily by the wave-wave interactions involving the synoptic-scale waves. On the other hand, in the studies of Cehelsky and Tung (1991) and Whitaker and Barcilon (1995) that use a two-layer quasigeostrophic model with a traditional asymmetric friction and a forcing that relaxes to a zonal atmospheric jet baroclinically unstable, it is found that the low-frequency variability draws its energy mainly from the wave-mean flow interactions.

\section{The present study}

The model we have used is the generic Phillips model briefly described in section 2 . One important difference of this study from other similar nonlinear studies is the length of the channel that is here large enough for both short waves and long waves (in terms of their zonal wavelengths with respect to the internal Rossby radius of deformation) to be potentially involved in the dynamics of the system. Thus, the nondimensional geometry of the channel considered is $0 \leq x \leq \gamma$ and 0 $\leq y \leq 1$ with $\gamma=4$ instead of $\gamma=2$ in the other studies. Since the channel is periodic in $x, \gamma$ is the period of the channel, that is, the largest zonal wavelength. So the comparison of our results with the other nonlinear studies allows us to better understand the effects of an asymmetric friction on the wave scale selection and the upscale energy transfer.

The parameter setting considered in this study is close to the one used by Mak $(1985,1987)$. The values are $F=27.6, \beta=31.4, \bar{r} / 2=\left(r_{1}+r_{2}\right) / 4=0.1$, and $U_{s}$ $=U_{1}=-U_{2}$ ranging from 0.56 to 1.27 . The minimum critical current shear $U_{c}$ is $0.53,0.41$, and 0.55 respectively for the symmetric, traditional and nontraditional asymmetric cases. So the chosen $U_{s}$ values correspond to a supercriticality [defined as $\Delta=\left(U_{s}-U_{c}\right) / U_{c}$ ] ranging from 0.018 to 2.1 . Thus, the baroclinic systems considered correspond to weakly $(\Delta \ll 1)$ to moderately $[\Delta=O(1)]$ unstable systems. Motivation of that choice is that nonlinearities are much simpler to explore and to understand when the supercriticality is not too large. Note that we have chosen to use $U_{s}$ as an independent forcing parameter instead of the supercriticality since the critical shear $U_{c}$ significantly depends on the degree of frictional asymmetry (see Fig. 1). The mean dissipation rate $(\bar{r} / 2)$ is lower than the values used by Mak (1987), and the degree of asymmetry, defined as $\alpha=$ $\left(r_{1}-r_{2}\right) /\left(r_{1}+r_{2}\right)$, has a large magnitude when the dissipation is asymmetric: it is -0.6666 and 0.6666 respectively for traditional $\left(r_{1}=0.2 r_{2}\right)$ and nontraditional $\left(r_{1}=5 r_{2}\right)$ asymmetric friction. This choice is based on the findings of previous studies and in particular that of Chou and Loesch (1986b), which showed that the effects of a viscous asymmetry on the nonlinear interactions are better displayed when the mean dissipation rate is low and the degree of asymmetry is large. However, the sensitivity of the results to the mean dissipation rate and to the degree of the asymmetry will be examined.

The spectral numerical method used to integrate Eqs. (1) and (2) is the one described in Klein and Pedlosky (1986). It is based on an appropriate spectral representation of the streamfunctions $\phi_{B}$ and $\phi_{T}$ that satisfies the boundary conditions

$$
\phi=\sum_{m} \sum_{n} A_{m n} e^{i m k x} \sin (n \pi y)+\sum_{p} A_{o p} \cos (p \pi y),
$$

with $k=2 \pi / \gamma, A_{m n}=A_{-m n}^{*},|m| \leq M$ with $m \neq 0,1 \leq$ $n \leq N$, and $1 \leq p \leq N M$. An asterisk denotes complex conjugation. Here $A_{m n}$ and $A_{o p}$ designate the Fourier components of the wave field and of the mean flow correction respectively. The spectral truncation used for most simulations is $M=N=N M=16$ or $M=N=$ 
TABle 1. Nonlinear equilibria obtained with $\overline{\mathrm{r}} / 2=0.1$ when the wave-wave interactions are suppressed. Here, $\mathbf{S}, \mathbf{P}$, and $\mathbf{C}$ respectively refer to steady, periodic and chaotic states; $(m, n)$ refers to the waves [identified by their zonal $(m)$ and meridional $(n)$ wavenumbers] whose amplitudes $\left(\left|A_{m n}\right|\right)$ dominate the nonlinear equilibria.

\begin{tabular}{|c|c|c|c|c|c|}
\hline & \multicolumn{5}{|c|}{$U_{S}$} \\
\hline & 0.56 & 0.63 & 0.79 & 0.95 & 1.27 \\
\hline $\begin{array}{l}\text { Symmetric dissipation } \\
\quad(\alpha=0)\end{array}$ & $\begin{array}{c}\mathbf{S} \\
(3,1)\end{array}$ & $\begin{array}{c}\mathbf{S} \\
(3,1)\end{array}$ & $\begin{array}{c}\mathbf{S} \\
(3,1)\end{array}$ & $\begin{array}{c}\mathbf{S} \\
(3,1)\end{array}$ & $\begin{array}{c}\mathbf{S} \\
(3,1)\end{array}$ \\
\hline $\begin{array}{l}\text { Nontraditional asymmetric dissipation } \\
\quad(\alpha=0.6666)\end{array}$ & & $\begin{array}{c}\mathbf{S} \\
(3,1)\end{array}$ & $\begin{array}{c}\mathbf{P} \\
(3,1)\end{array}$ & $\begin{array}{c}\mathbf{P} \\
(3,1)\end{array}$ & $\begin{array}{c}\mathbf{C} \\
(3,1) \\
(2,1)\end{array}$ \\
\hline $\begin{array}{l}\text { Traditional asymmetric dissipation } \\
\quad(\alpha=-0.6666)\end{array}$ & $\begin{array}{c}\mathbf{S} \\
(1,2)(4,1)\end{array}$ & $\begin{array}{c}\mathbf{S} \\
(1,2) \\
(3,1)\end{array}$ & $\begin{array}{c}\mathbf{S} \\
(1,2) \\
(3,1)\end{array}$ & $\begin{array}{c}\mathbf{S} \\
(1,2) \\
(3,1)\end{array}$ & $\begin{array}{c}\mathbf{C} \\
(1,2)(3,1) \\
(2,1)(2,2)\end{array}$ \\
\hline
\end{tabular}

$N M=32$. Main results have been checked with a spectral truncation corresponding to $M=N=N M=50$. Sensitivity of the results to the higher-order stable modes is discussed in section 4c. In the following, waves are identified by their zonal and meridional wavenumbers $(m, n)$. For instance, $(1,2)$ is a wave with a long zonal wavelength and a meridional structure that is a dipole, whereas $(3,1)$ is a wave with a short zonal wavelength and a large meridional extension.

A linear stability analysis for $\vec{r} / 2=0.1$ and $U_{s}$ ranging from 0.56 to 1.27 has been performed. When $U_{s}=0.56$ (i.e., for a weakly unstable system), $(3,1)$ is the most unstable wave in the symmetric and nontraditional asymmetric cases whereas in the traditional asymmetric case $(1,2)$ is the most unstable wave (see Fig. 2). However, for $U_{s}$ ranging from 0.63 to 1.27 , the results (not shown) do not reveal any significant differences due to the asymmetry: The wave $(3,1)$ is the most unstable one (see Fig. 3 for $U_{s}=0.95$ ). Thus, for most of the $U_{s}$ value considered, the presence and the sense of an asymmetric friction has no effect on the most linearly unstable waves. It has an effect (leading in particular to a wave scale selection) only for one $U_{s}$ value $\left(U_{s}=\right.$ 0.56 ) corresponding to a weakly unstable baroclinic system.

\section{Nonlinear results}

Several numerical simulations have been performed with $U_{s}$ varying between 0.56 and 1.27 and the mean dissipation rate held constant $(\bar{r} / 2=0.1$, which corresponds to an $e$-folding time of 10 nondimensional time units) as a guideline. Although the linear stability analysis does not display any significant differences due to the presence of a viscous asymmetry for $U_{s} \geq 0.63$, the nonlinear results do reveal that the presence and sense of the viscous asymmetry has a significant influence on the nonlinear equilibria and, in particular, induces a strong wave scale selection. This implies a significant effect of an asymmetric friction on the nonlinear interactions, that is, the wave-wave and wave-mean flow interactions.

The fully nonlinear simulations have revealed that with a nonzero frictional asymmetry the nonlinear equilibria are mostly dominated by one wave, which means that the wave-wave interactions are quite weak. So, in order to determine the respective role of the wave-mean flow and wave-wave interactions in the setup of these equilibria, we have performed another series of simulations with the wave-wave interactions suppressed to compare with the fully nonlinear simulations. This series of simulations clearly shows that the wave-mean flow interactions alone are able to significantly induce this wave scale selection. Then, in order to better emphasize the respective role of the wave-mean flow and the wave-wave interactions, results with the wave-wave interactions suppressed are first presented in the next section. The nonlinear equilibrations are described in terms of the characteristics of the spatial and temporal structures of the total streamfunction and in particular of the dominant waves. The temporal evolution is characterized by the index of vacillation (defined as the ratio of the maximum amplitude variation to the mean amplitude) and the frequency spectrum of the dominant waves. When the index of vacillation is larger than 0.2 , the temporal evolution is labeled as periodic or chaotic, depending on whether the frequency spectrum is discrete or continuous. Otherwise, the temporal evolution is labeled as steady or quasisteady (when affected by weak oscillations). In most of the simulations these nonlinear equilibria are attained after an intermediate stage of about 250 nondimensional time units, which is quite large compared to the dissipation timescale $(\approx 10$ time units).

\section{a. With wave-mean flow interactions only}

The Jacobian terms in (1) and (2), have the form

$$
J(a, b)=J\left(a^{\prime}, \bar{b}\right)+J\left(\bar{a}, b^{\prime}\right)+J\left(a^{\prime}, b^{\prime}\right),
$$

where $\bar{a}$ denotes the zonal mean and $a^{\prime}=a-\bar{a}$. Thus, $J\left(a^{\prime}, b^{\prime}\right)$ represents the wave-wave interactions and $J(a$, $b)-J\left(a^{\prime}, b^{\prime}\right)$ the wave-mean flow interactions. Results reported in this section concern simulations performed with $J\left(a^{\prime}, b^{\prime}\right)=0$ and are summarized in Table 1 . The most important feature that comes out is the strong wave 
TABle 2. Same as Table 1 but when both the wave-mean flow and wave-wave interactions are included. Here, QS refers to a quasisteady state.

\begin{tabular}{|c|c|c|c|c|c|}
\hline & \multicolumn{5}{|c|}{$U_{S}$} \\
\hline & 0.56 & 0.63 & 0.79 & 0.95 & 1.27 \\
\hline $\begin{array}{l}\text { Symmetric dissipation } \\
\qquad(\alpha=0)\end{array}$ & $\begin{array}{c}\mathbf{S} \\
(1,2)\end{array}$ & $\begin{array}{c}\mathbf{P} \\
(1,2) \\
(4,1)(3,1)\end{array}$ & $\begin{array}{c}\text { QS } \\
(3,1) \\
(2,2)(1,1)\end{array}$ & $\begin{array}{c}\text { QS } \\
(3,1) \\
(2,2)(1,1)\end{array}$ & $\begin{array}{c}\mathbf{C} \\
(3,1)(1,1)(2,2) \\
(2,1)(1,2)(4,1)\end{array}$ \\
\hline $\begin{array}{l}\text { Nontraditional asymmetric dissipation } \\
\quad(\alpha=0.6666)\end{array}$ & & $\begin{array}{c}\mathbf{P} \\
(3,1)\end{array}$ & $\begin{array}{c}\mathbf{P} \\
(3,1)\end{array}$ & $\begin{array}{c}\mathbf{C} \\
(3,1)\end{array}$ & $\begin{array}{c}\mathbf{C} \\
(3,1)\end{array}$ \\
\hline $\begin{array}{l}\text { Traditional asymmetric dissipation } \\
\qquad(\alpha=-0.6666)\end{array}$ & $\begin{array}{c}\text { QS } \\
(1,2) \\
(4,1)\end{array}$ & $\begin{array}{c}\text { QS } \\
(1,2)\end{array}$ & $\begin{array}{c}\text { QS } \\
(1,2)\end{array}$ & $\begin{array}{c}\text { QS } \\
(1,2)\end{array}$ & $\begin{array}{c}\mathbf{C} \\
(1,1)(3,1)(2,2) \\
(1,2)(2,1)\end{array}$ \\
\hline
\end{tabular}

scale selection induced by the sense of the asymmetric friction.

In the symmetric and nontraditional asymmetric cases the most linearly unstable wave $(3,1)$ is the one that dominates all the nonlinear equilibria. For most $U_{s}$ values, the nontraditional frictional asymmetry makes the $(3,1)$ amplitude small and its time behavior destabilized. The nonlinear equilibria that emerge when the sense of the asymmetry is reversed, that is, in the traditional asymmetric case, completely differ from the previous ones: the spectral band of the dominant waves is larger and mostly the wave $(1,2)$, that is, with the longest zonal wavelength, dominates all the nonlinear equilibria. For $U_{s}=0.56$, waves $(1,2)$ and $(4,1)$ have almost an equal amplitude, but for $0.63 \leq U_{s} \leq 0.95,(1,2)$ has an amplitude at least twice as large as $(3,1)$. For $U_{s}=1.27$, the spectral band of the emerging waves is larger but $(1,2)$ still dominates.

It is important to stress that in the three dissipative cases the correction of the mean zonal flow, well dominated by its first spectral component, is such that the total baroclinic shear at the middle of the channel is almost equal to the critical shear. Furthermore, the presence of a nonzero frictional asymmetry induces a significant barotropic zonal jet whose amplitude satisfies (3), which confirms the findings of Pedlosky (1983) and Mak (1987).

\section{b. With the wave-wave interactions included}

The nonlinear equilibrations obtained with the full nonlinear interactions taken into account are summarized in Table 2. We have found that in the three dissipative cases the mean flow correction has again its baroclinic part dominated by the first spectral component and the total baroclinic shear at the middle of the channel is slightly larger than the critical value. Furthermore, the asymmetric cases are again characterized by the presence of a significant barotropic zonal jet whose amplitude almost satisfies (3). Comparison of Table 2 with Table 1 gives some information about the effects of the wave-wave interactions in each dissipative case.

\section{1) THE SYMMETRIC CASE}

The larger spectral band of the dominant waves displayed in Table 2 (compared with Table 1) emphasizes the role of the wave-wave interactions in the upscale energy transfer. Let us examine how these wave-wave interactions work.

When $U_{s} \leq 0.63$, the wave-wave interactions make the wave $(1,2)$ become dominant. We have found that this wave is selected mainly by the triads $\{(3,1),(2$, $1),(1,2)\}$ and $\{(5,1),(4,1),(1,2)\}$ that work only during an intermediate stage when $U_{s}=0.56$ and that alternately resonate at equilibrium when $U_{s}=0.63$ (a triad is resonant when the sum of the phases of the three wave modes is time independent). For $U_{s}$ ranging from 0.79 to 1.27 , the wave-wave interactions mostly concern the triad $\{(3,1),(2,2),(1,1)\}$, which makes the waves $(2,2)$ and $(1,1)$ emerge significantly with wave $(1,1)$ having a very strong barotropic structure: the ratio of its barotropic amplitude to its baroclinic amplitude is equal to 5 instead of 2 as for the other waves. For $U_{s}=0.95$, this triad is almost permanently resonant (Fig. 4a), which in addition to the quasi-steady state of the wave amplitudes (Fig. 5) indicates that the energy transfer among the waves and between the waves and the mean flow is quasi steady. When $U_{s}=1.27$, this triad is intermittently resonant (Fig. 4b), which in addition to the high-frequency fluctuations of the wave amplitudes (Fig. 6) indicates that the energy transfer among the waves is strongly time varying. However, the energy transfer between the waves and the mean flow is almost constant since the mean flow evolution is quasi steady (Fig. 6). These features have been confirmed by an energy budget analysis (Rivière 1995). An illustration of the flow regimes obtained in these nonlinear equilibria is shown in Fig. 7. It corresponds to a circulation with a strong zonal flow perturbed by energetic short waves and weaker longer waves.

\section{2) The NONTRAditional ASYMmETRIC CASE}

The remarkable characteristics of the results in this case is that the nonlinear equilibria are almost close to the ones obtained when the wave-wave interactions are 

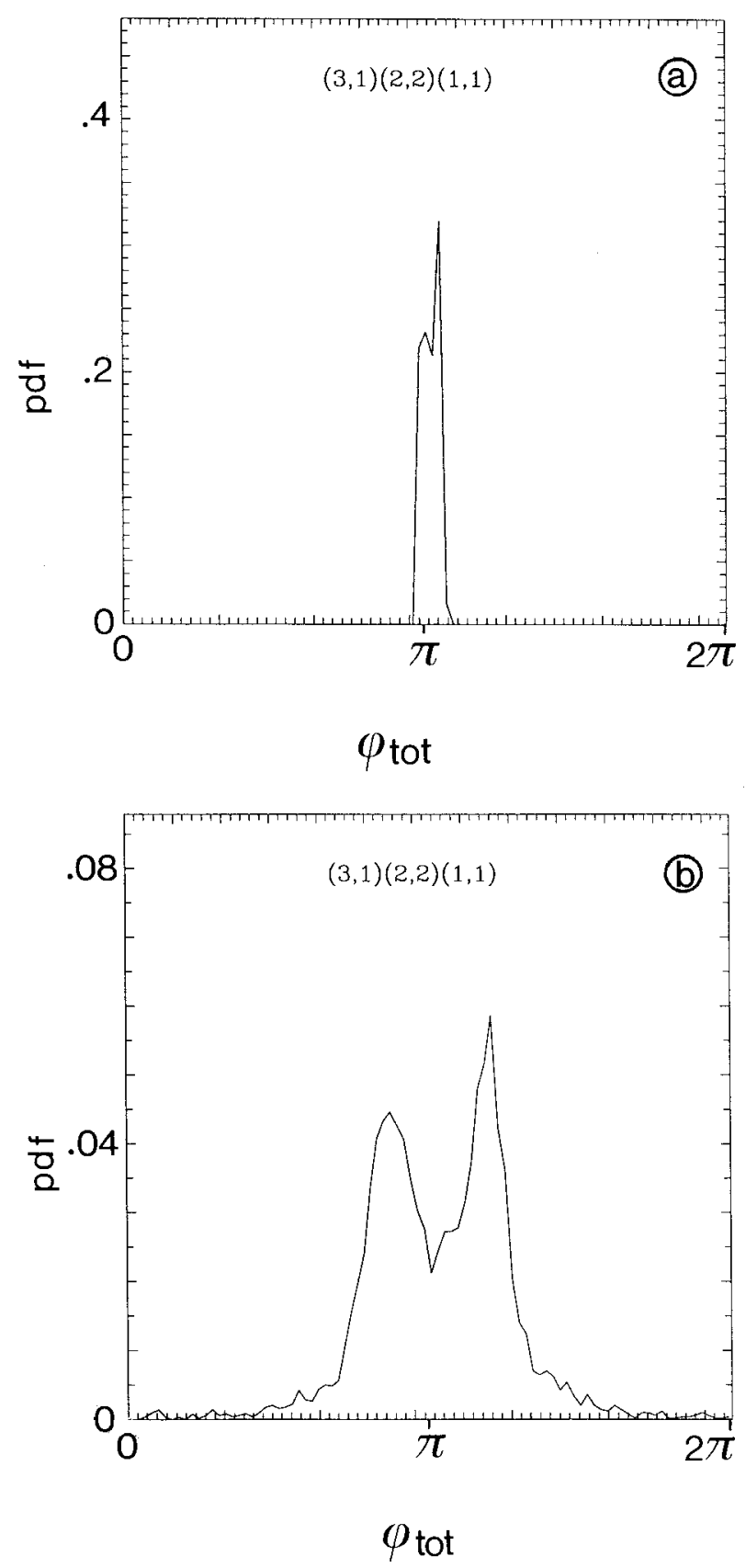

FIG. 4. Probability density function (pdf) of the spatial phase relationship of the triad $\{(3,1),(2,2),(1,1)\}\left[\varphi_{\mathrm{tot}}=\varphi_{31}^{T}+\varphi_{22}^{T}+\right.$ $\varphi_{11}^{B}$, with $\varphi_{m n}^{B, T}$ the phase of the barotropic $(B)$ or the baroclinic part (T) of the wave $(m, n)]$ when (a) $U_{s}=0.95$ and (b) $U_{s}=1.27$ in the symmetric case. The strong bimodal structure displayed in (b) is the signature of the significant high-frequency fluctuations that affect $\varphi_{\text {tot }}$ when $U_{s}=1.27$.

suppressed (cf. Tables 1 and 2); $(3,1)$ is the only wave to emerge for all $U_{s}$ values. Thus, the effects of the wave-wave interactions in terms of energy transfer between the waves appear to be strongly inhibited. We have found, however, a very weak activation of these wave-wave interactions, which makes the time evolu- tion of the wave $(3,1)$ become destabilized. When $U_{s}$ $=1.27$, the wave-wave interactions act to remove energy from wave $(2,1)$ [that was present when $J\left(a^{\prime}, b^{\prime}\right)$ $=0]$ to the benefit of $(3,1)$. One particular feature revealed by the equilibrium for this large $U_{s}$ value is the intermittent appearance of wave $(1,1)$ characterized by very small peaks (Fig. 8). This is due to the triad $\{(1,1),(3,1),(2,2)\}$, which is resonant only during a short period when the $(3,1)$ amplitude attains a large value. So even for this large $U_{s}$ value the wave-wave interactions appear to be unable to efficiently transfer energy among the waves. In Fig. 9, the flow regime in this dissipative case is characterized by a zonal flow that is stronger than in the symmetric case.

\section{3) The traditional ASYMMETRIC CASE}

Completely different nonlinear equilibria are obtained in this dissipative case. For $U_{s}$ ranging from 0.56 to 0.95 (Table 2), they are characterized by the emergence of a single wave with the longest zonal wavelength: $(1,2)$ (see Fig. 10). An energy budget analysis performed in the intermediate stage has confirmed the important role of the wave-mean flow interactions in the wave scale selection and the specific action of the wave-wave interactions in the reinforcement of this scale selection (Rivière 1995). This reinforcement is well revealed by the comparison of Table 2 with Table 1, which shows that the presence of the wave-wave interactions make the wave $(4,1)$ decrease (by a factor of 2) when $U_{s}=$ 0.56 , while they reduce $(3,1)$ amplitude (by a factor of 6 ) for other $U_{s}$ values. Furthermore, the $(1,2)$ amplitude is slightly increased (by a factor of 1.2). So, with a traditional frictional asymmetry, the wave-wave interactions strongly promote the emergence of the long (in terms of its zonal wavelength) wave $(1,2)$ at the expense of the short waves $(3,1)$ and $(4,1)$. These wave-wave interactions are most efficient during an intermediate stage so that, at the equilibrium, $(1,2)$ is the only wave present. Figure 11 shows an illustration of the flow regime obtained in this dissipative case; the pattern does not look like a zonal regime but is more like a blocking regime.

When $U_{s}=1.27$, the nonlinear equilibration is characterized by a large spectral wave band and a chaotic time evolution. For this forcing the role of the wavewave interactions appears to be similar, although their effects are not so pronounced; their presence decreases the $(3,1)$ amplitude by a factor of 1.5 and wave $(1,1)$ becomes dominant. The wave $(1,2)$ amplitude is, however, slightly reduced. We have found that the triad $\{(1$, $1),(3,1),(2,2)\}$ is resonant and drives the energy transfer to $(1,1)$.

\section{c. Discussion}

With only the wave-mean flow interactions included, the presence and sense of an asymmetric friction induces 

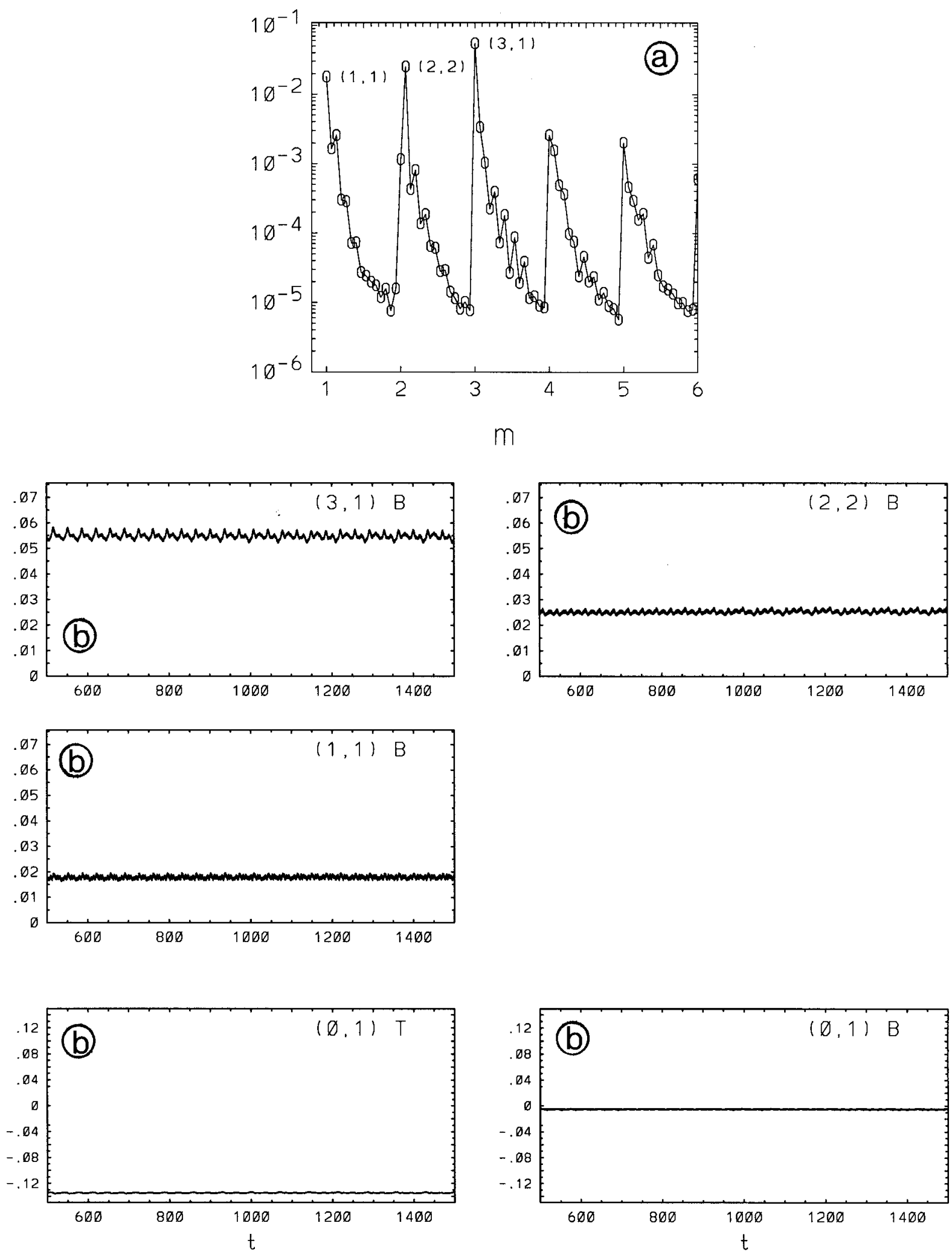

FIG. 5. (a) Time mean (averaged over 1000 time units) barotropic amplitude spectrum of the wave field when $U_{\mathrm{s}}=0.95$ in the symmetric case. Labels on the horizontal axis refer to the zonal wavenumber. Meridional wavenumber varies from 1 to 16 between two successive values of the zonal wavenumber. Dominant waves are indicated. (b) Time evolution of the modulus of the barotropic amplitude of the dominant waves $(m, n)^{B}$, and of the baroclinic $(0,1)^{T}$ and barotropic $(0,1)^{B}$ amplitude of the mean flow correction. 


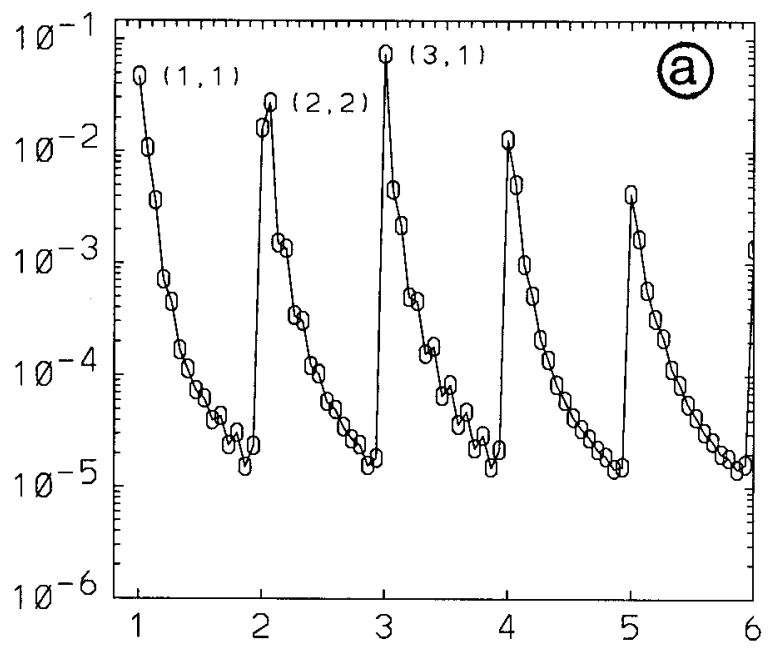

m
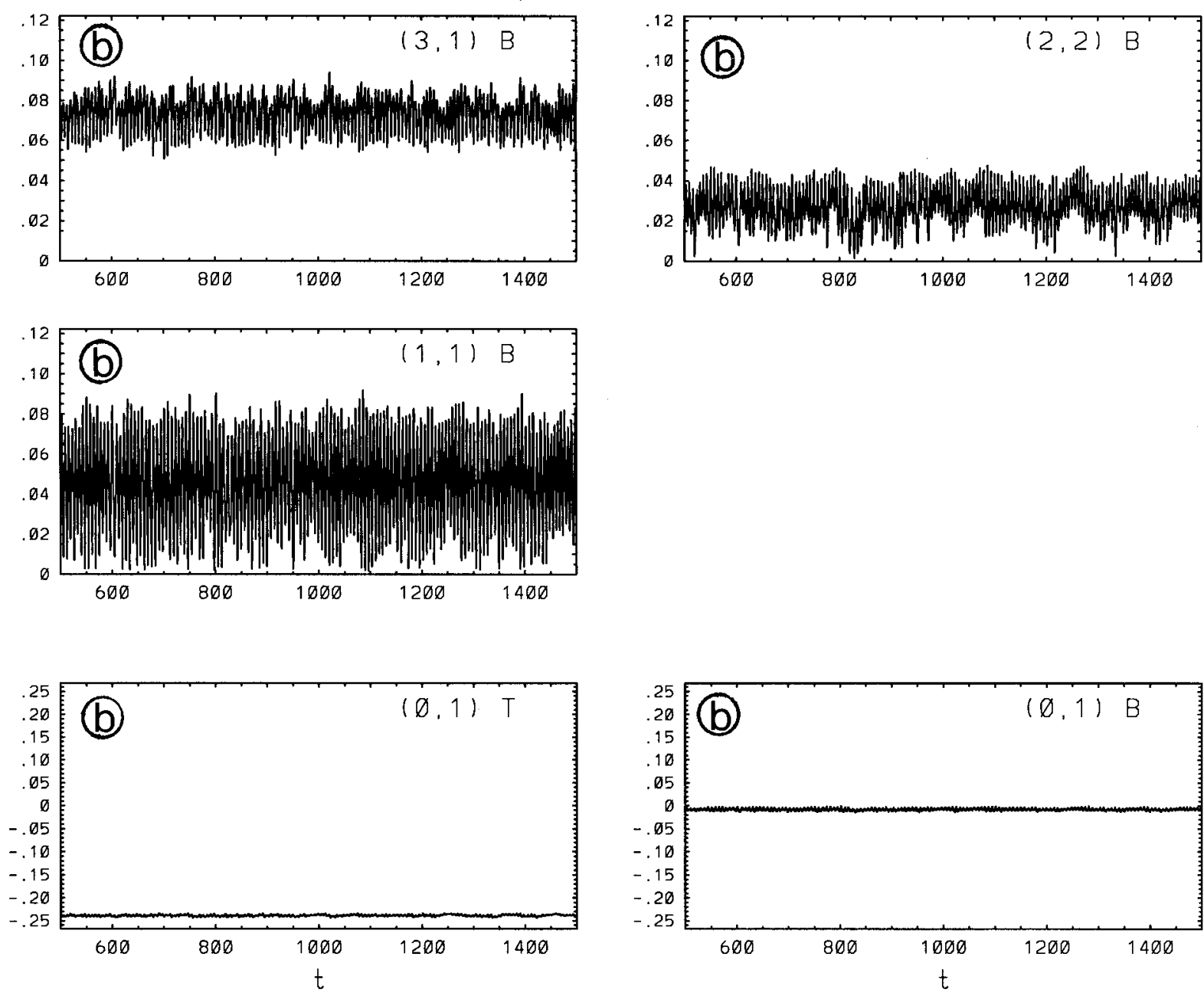

Fig. 6. As in Fig. 5 but when $U_{s}=1.27$. 


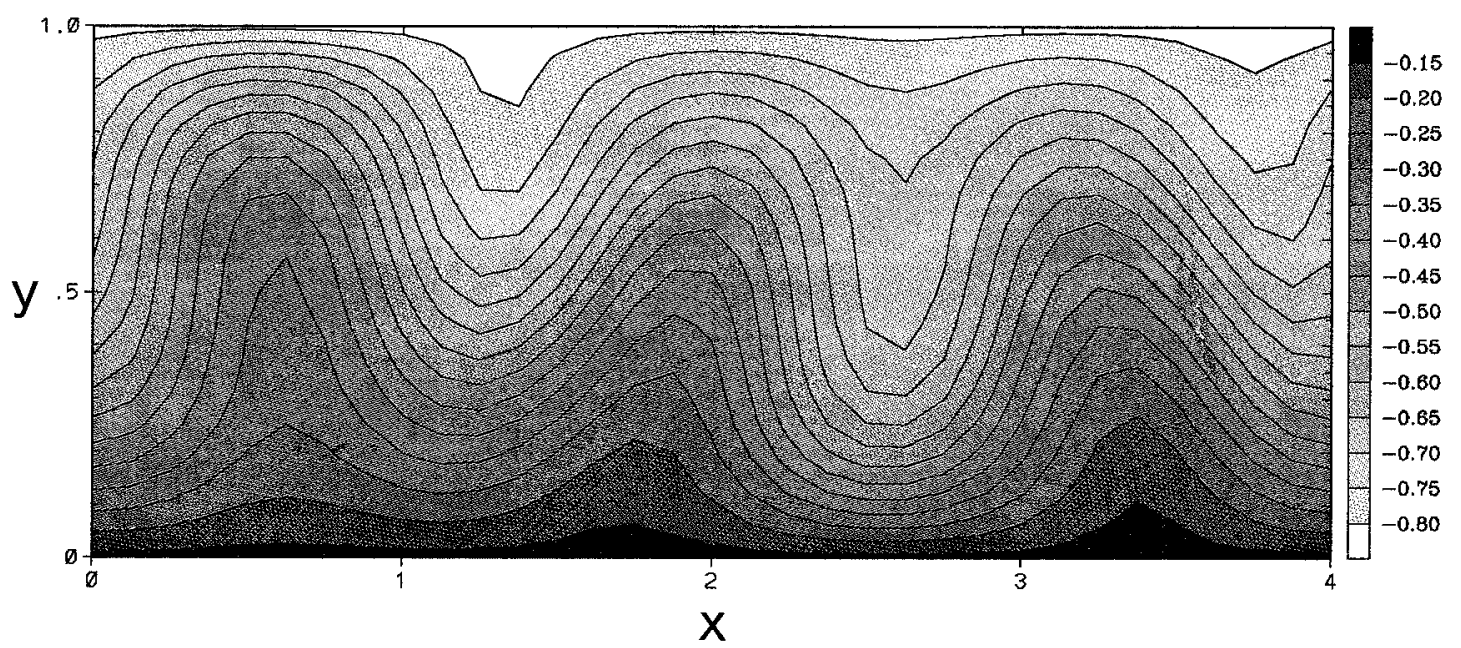

Fig. 7. Snapshot of the upper level total streamfunction at $t=1200$ when $U_{s}=0.95$ in the symmetric case.

a strong wave scale selection. While only one wave emerges in the symmetric case, several waves emerge in the traditional asymmetric case (for all $U_{s}$ values) and in the nontraditional case (for $U_{s}=1.27$ ). The main noteworthy feature is observed with a traditional asymmetric friction: the dominant wave has a much longer zonal wavelength than in the other frictional cases. It is interesting to note that in this asymmetric case this wave, which is only the third and the fifth most linearly unstable wave for $U_{s}=0.79$ and $U_{s}=0.95$ respectively, is the first most unstable one when $U_{s}=0.56$, that is, when the system is weakly unstable (see Fig. 2). Let us stress again that in the three dissipative cases the correction of the mean zonal flow is such that the total baroclinic shear at the middle of the channel is almost equal to the critical shear.

Inclusion of the wave-wave interactions strongly increases the differences between the three dissipative cases and, in particular, reinforces the wave scale selection. In the symmetric case the wave-wave interactions enlarge the spectral band of the dominant waves (with both long and short zonal wavelengths). This result particularly emphasizes their classic role in upscale energy transfer. In the traditional asymmetric case the wave-wave interactions reduce the spectral wave band of the dominant waves when supercriticality is not too large. In that case they appear to induce a pronounced unidirectional energy transfer from the short unstable waves to the longer waves: the long wave $(1,2)$, mainly selected by the wave-mean flow interactions, is reinforced by the wave-wave interactions. In the nontraditional asymmetric case, the effects of the wave-wave interactions, in terms of energy transfer among the waves and, in particular, in the upscale energy transfer, are strongly inhibited.

We have examined the sensitivity of these nonlinear equilibria to the numerical resolution. Simulations performed with $M=N=N M \geq 16$ produce the same results. When $M, N, N M<16$, some differences appear that emphasize the sensitivity of the nonlinear equilibria to the higher-order harmonics and in particular to the meridional harmonics. For example, whereas the equilibrium obtained with $M=3, N=7, N M=15$ in the traditional asymmetric case (with $U_{s}=0.95$ ) does not differ much from the one with a high resolution, the equilibrium obtained with $M=N=3, N M=15$ displays a large spectral wave band including $(1,2)$ and $(2,2)$ with the same amplitude and $(3,1)$ and $(1,1)$ with an amplitude only two times smaller (Rivière 1995). Thus, the meridional harmonics have a significant impact on the wave-wave interactions. This impact of the higher-order harmonics seems to be related to the aspect ratio of the channel. Indeed, when the channel zonal length is smaller, using for example $\gamma=2$ (such that only short waves can develop), we have found that the higher-order harmonics of the dominant waves have no effect on the nonlinear equilibrium, which well confirms Mak's (1987) results. This means that, when the system allows the emergence of both short and long waves (in terms of their zonal wavelength), the role of the higher-order harmonics of the dominant waves and, in particular, of the meridional harmonics becomes important for the energy transfer between these waves.

Thus it is clear, at least for the parameter setting chosen in this study, that the presence and the sense of a frictional asymmetry significantly affect the nonlinear interactions, which leads to a significant wave scale selection that affects both the wave scales and the number of the dominant waves that emerge in the equilibrium.

\section{Sensitivity to the mean dissipation rate and to the degree of asymmetry}

\section{a. Sensitivity to the mean dissipation rate}

The results presented so far have been obtained with the parameter $\bar{r} / 2$ held constant as a guideline. Addi- 


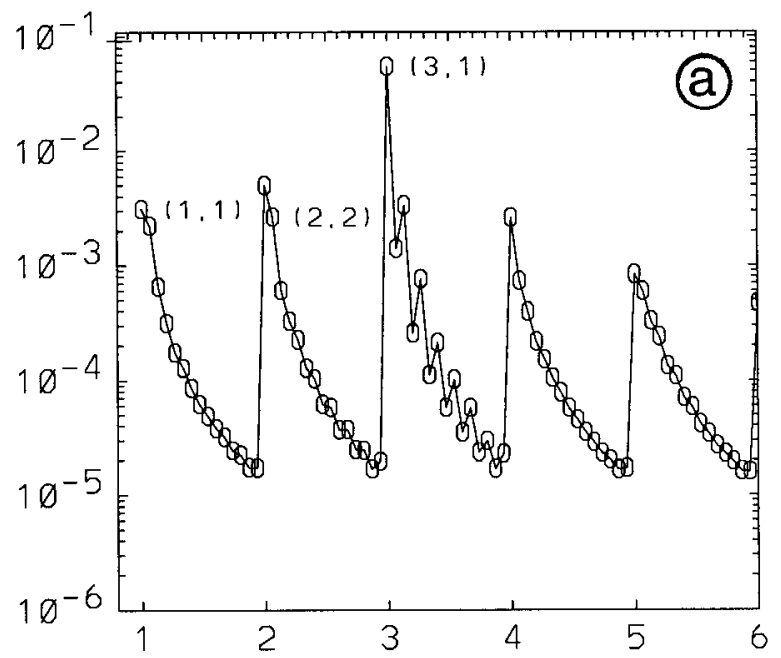

m
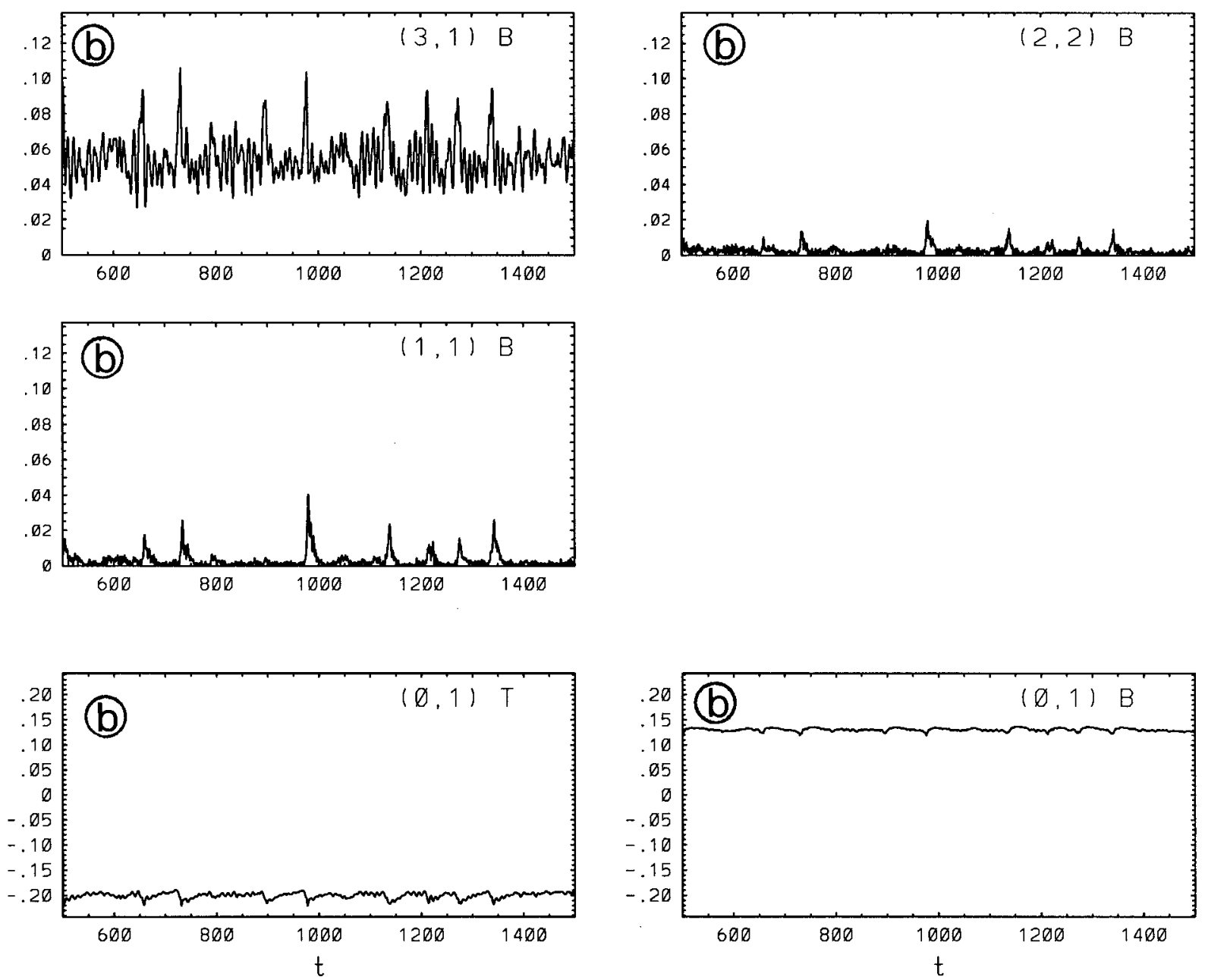

FIG. 8. As in Fig. 5 but when $U_{s}=1.27$ in the nontraditional asymmetric case. 


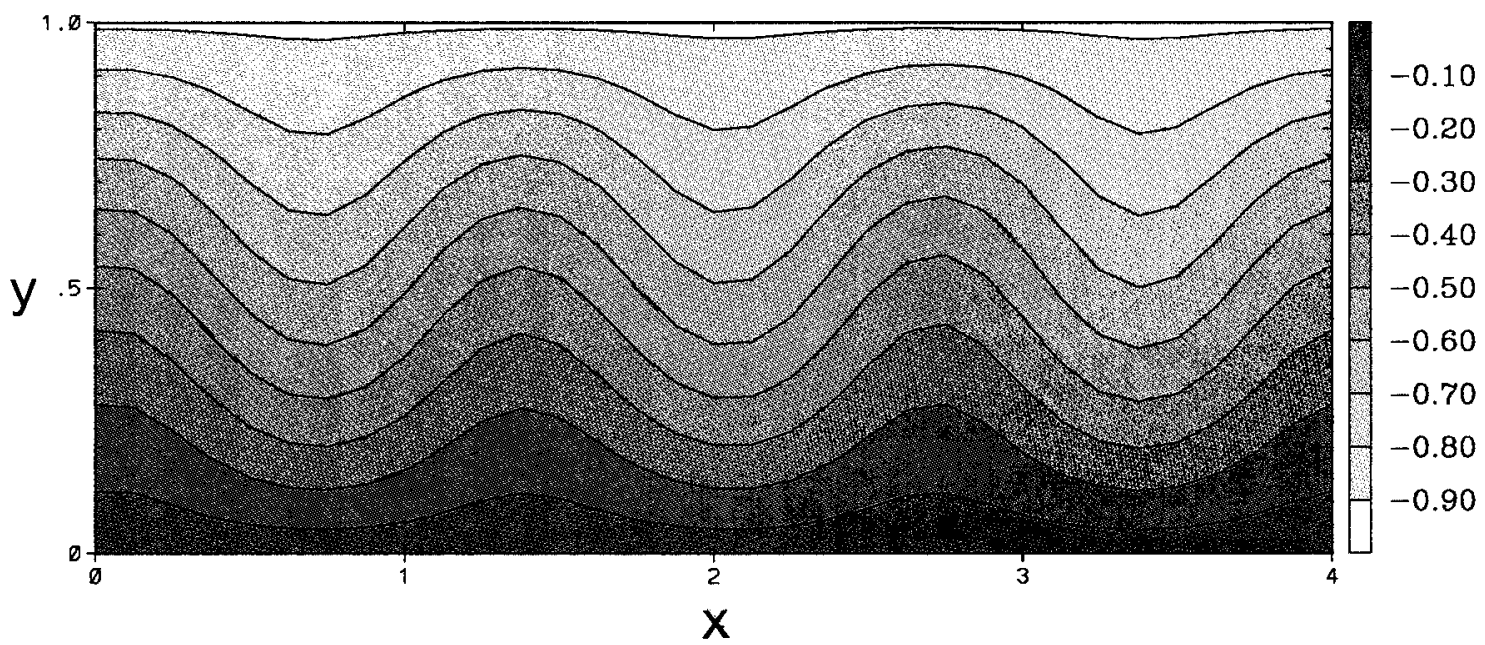

FIG. 9. Snapshot of the upper level total streamfunction at $t=1200$ when $U_{s}=0.95$ in the nontraditional asymmetric case.

tional numerical simulations have been performed to examine their robustness with $U_{s}=0.95$ and $\bar{r} / 2$ varying. The degrees of asymmetry considered are the same as before. The results displayed in Table 3 are qualitatively close to the ones obtained with $U_{s}=0.95$ and $\bar{r} / 2=0.1$, which means that the results obtained pre- viously are robust when the mean dissipation rate $\bar{r} / 2$ is changed.

The main new result concerns the traditional asymmetric case for which a large mean dissipation $(\bar{r} / 2=$ 0.35 ) appears to strongly activate some specific wavewave interactions. Indeed, the corresponding equilib-
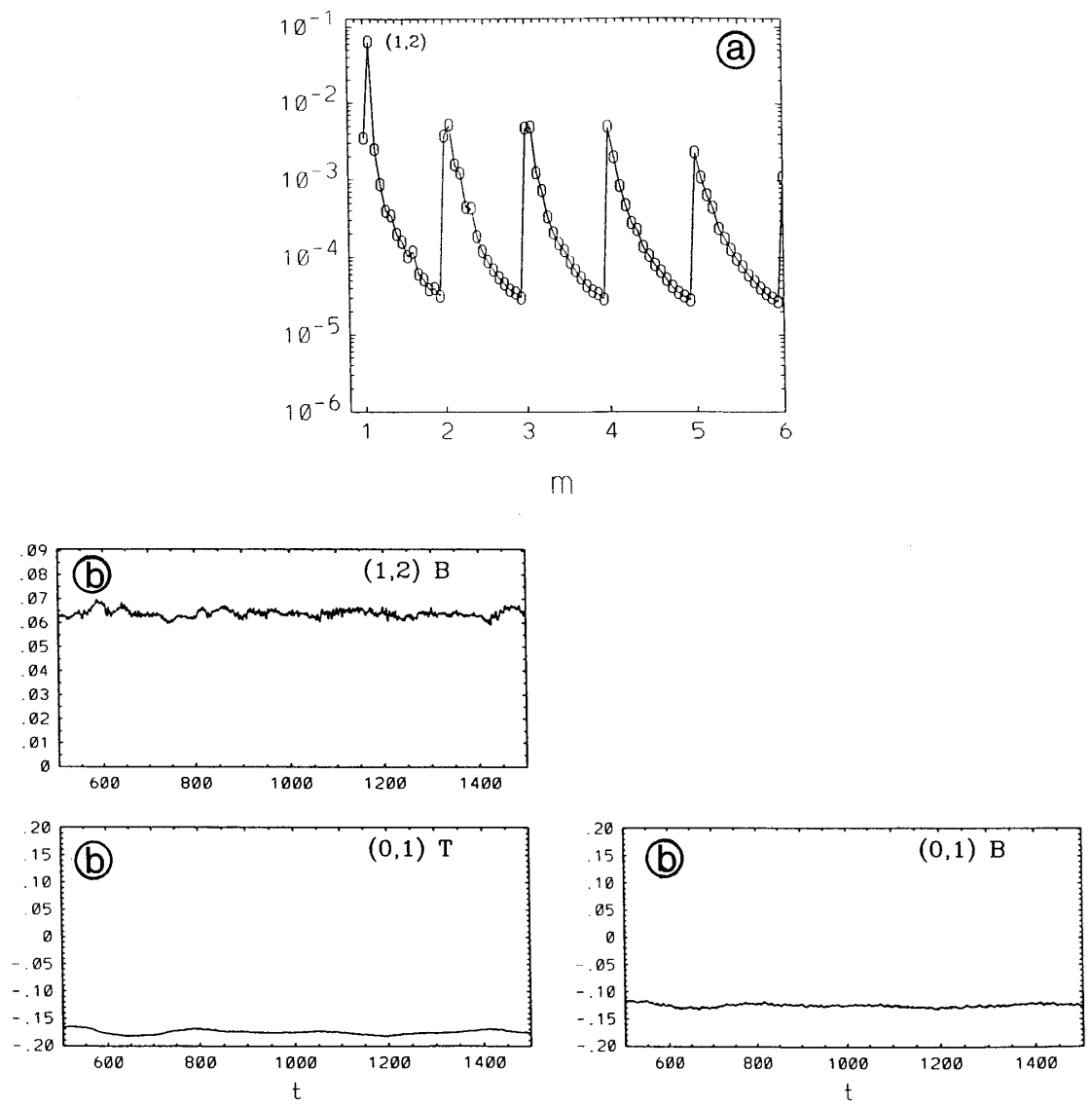

FIG. 10. As in Fig. 5 but when $U_{s}=0.95$ in the traditional asymmetric case. 
TABLE 3. Same as Table 2 but with $U_{S}=0.95$ and $\overline{\mathrm{r}} / 2$ varying.

\begin{tabular}{|c|c|c|c|c|c|c|}
\hline & \multicolumn{6}{|c|}{$\overline{\mathrm{r}} / 2$} \\
\hline & 0.02 & 0.07 & 0.1 & 0.14 & 0.21 & 0.35 \\
\hline $\begin{array}{l}\text { Symmetric dissipation } \\
\quad(\alpha=0)\end{array}$ & $\begin{array}{c}\mathbf{C} \\
(3,1)(4,1) \\
(1,1)(1,2)\end{array}$ & $\begin{array}{c}\mathbf{P} \\
(3,1)(4,1) \\
(1,1)(1,2)\end{array}$ & $\begin{array}{c}\mathbf{Q S} \\
(3,1) \\
(2,2)(1,1)\end{array}$ & $\begin{array}{c}\mathbf{S} \\
(3,1) \\
(2,2)(1,1)\end{array}$ & $\begin{array}{c}\mathbf{S} \\
(3,1) \\
(2,2)(1,1)\end{array}$ & $\begin{array}{c}\mathbf{S} \\
(3,1)\end{array}$ \\
\hline $\begin{array}{l}\text { Nontraditional asymmetric dissipation } \\
\quad(\alpha=0.6666)\end{array}$ & $\begin{array}{c}\mathbf{C} \\
(3,1) \\
(2,2)(1,1)\end{array}$ & $\begin{array}{c}\mathbf{C} \\
(3,1)\end{array}$ & $\begin{array}{c}\mathbf{C} \\
(3,1)\end{array}$ & $\begin{array}{c}\mathbf{C} \\
(3,1)\end{array}$ & $\begin{array}{c}\mathbf{C} \\
(3,1)\end{array}$ & $\begin{array}{c}\mathbf{C} \\
(3,1)\end{array}$ \\
\hline $\begin{array}{l}\text { Traditional asymmetric dissipation } \\
\quad(\alpha=-0.6666)\end{array}$ & $\begin{array}{l}\mathbf{Q S} \\
(1,2)\end{array}$ & $\begin{array}{l}\mathbf{Q S} \\
(1,2)\end{array}$ & $\begin{array}{l}\mathbf{Q S} \\
(1,2)\end{array}$ & $\begin{array}{l}\text { QS } \\
(1,2)\end{array}$ & $\begin{array}{c}\text { QS } \\
(1,2)\end{array}$ & $\begin{array}{c}\mathbf{C} \\
(2,2)(3,1) \\
(1,1)(1,2)\end{array}$ \\
\hline
\end{tabular}

rium, quite different from the ones obtained with smaller $\bar{r} / 2$ values, looks similar to those observed in the symmetric case for smaller dissipation values. Clearly there is a bifurcation point for an $\bar{r} / 2$ value between 0.21 and 0.35 that makes the wave-wave interactions and, in particular, the triad $\{(3,1),(2,2),(1,1)\}$ strongly activated. On the other hand, variation of the mean dissipation rate in the symmetric case nicely emphasizes the classic role of the friction on the wave-wave interactions; this activity (revealed by the destabilization of the time behavior and the amplitude of the long waves relative to the short ones) is enhanced when the dissipation is reduced while the opposite tendency is observed when the mean dissipation rate is increased. In the nontraditional case the nonlinear equilibria are almost insensitive to the variation of $\bar{r} / 2$. Only for $\bar{r} / 2=0.02$ does a weak activity of the triad $\{(3,1),(2,2),(1,1)\}$ make waves $(2,2)$ and $(1,1)$ emerge [with amplitudes respectively 3 and 6.5 times smaller than $(3,1)$ amplitude].

\section{b. Sensitivity to the degree of asymmetry}

Sensitivity of the characteristics of the nonlinear equilibria to the degree of the asymmetry has been examined with $U_{s}=0.95$ and $\bar{r} / 2=0.1$. The results (Table 4) mostly reveal that a large degree of asymmetry is required for a frictional asymmetry to significantly affect the nonlinear equilibrium of a baroclinic system.

In the traditional asymmetric case, the characteristics of the equilibrium resemble the ones of the symmetric case when the asymmetry is reduced: when $\alpha \geq-0.5$, the triad $\{(3,1),(2,2),(1,1)\}$ is strongly activated. The wave $(1,2)$ amplitude is five times smaller than $(3$, 1 ) for $\alpha=-0.5$ and negligible for $\alpha=-0.2$. When the asymmetry is large (i.e., for $\alpha<-0.6666$ ), wave $(1,2)$ is the dominant one, although its amplitude decreases as the asymmetry increases. In the nontraditional asymmetric case the results found for $\alpha=0.6666$ are robust for a much larger range of the degree of asymmetry. Only for $\alpha=0.2$ does the equilibrium differ and is now close to the one observed in the symmetric case with waves $(3,1),(2,2)$, and $(1,1)$ dominating and the time behavior being quasi steady. Note that the results in the nontraditional asymmetric case follow the ones reported by Mak (1987): chaotic behavior is observed only when the asymmetry becomes large.

In all simulations, we have found again that the mean flow correction involves a nonzero barotropic part that satisfies the relation (3). Lastly, it is interesting to note that with a stress-free upper or lower boundary ( $\alpha=$ $\pm 1)$ the equilibrium is almost a wave-free state. This reveals that the results of Pedlosky (1983) and Chou and Loesch (1986b) about the nonlinear equilibration of a baroclinic system with a stress-free upper boundary are still robust for a moderately unstable system in the $\beta$ plane. Using the argument of Pedlosky (1983) and that our equilibria (with $|\alpha|<1$ ) usually involve only one wave, this result is understandable; for example, when $\alpha=-1$, there is no mean potential vorticity dis-

TABLE 4. Same as Table 2 but with $U_{S}=0.95, \overline{\mathrm{r}} / 2=0.1$, and the degree of asymmetry $\alpha=\left(r_{1}-r_{2}\right) /\left(r_{1}+r_{2}\right)$ varying.

\begin{tabular}{|c|c|c|c|c|c|}
\hline & \multicolumn{5}{|c|}{$\alpha$} \\
\hline & -1 & -0.9355 & -0.6666 & -0.5 & -0.2 \\
\hline \multirow[t]{3}{*}{ Traditional asymmetric dissipation } & $\begin{array}{l}\text { zonal } \\
\text { flow }\end{array}$ & $\begin{array}{c}\mathbf{C} \\
(1,2)(2,2)\end{array}$ & $\begin{array}{c}\text { QS } \\
(1,2)\end{array}$ & $\begin{array}{c}\mathbf{C} \\
(3,1) \\
(2,2)(1,1)\end{array}$ & $\begin{array}{c}\text { QS } \\
(3,1) \\
(2,2)(1,1)\end{array}$ \\
\hline & \multicolumn{5}{|c|}{$\alpha$} \\
\hline & 0.2 & 0.5 & 0.6666 & 0.9355 & 1 \\
\hline Nontraditional asymmetric dissipation & $\begin{array}{c}\text { QS } \\
(3,1) \\
(2,2)(1,1)\end{array}$ & $\begin{array}{c}\text { C } \\
(3,1)\end{array}$ & $\begin{array}{c}\mathbf{C} \\
(3,1)\end{array}$ & $\begin{array}{c}\mathbf{C} \\
(3,1)\end{array}$ & $\begin{array}{l}\text { zonal } \\
\text { flow }\end{array}$ \\
\hline
\end{tabular}




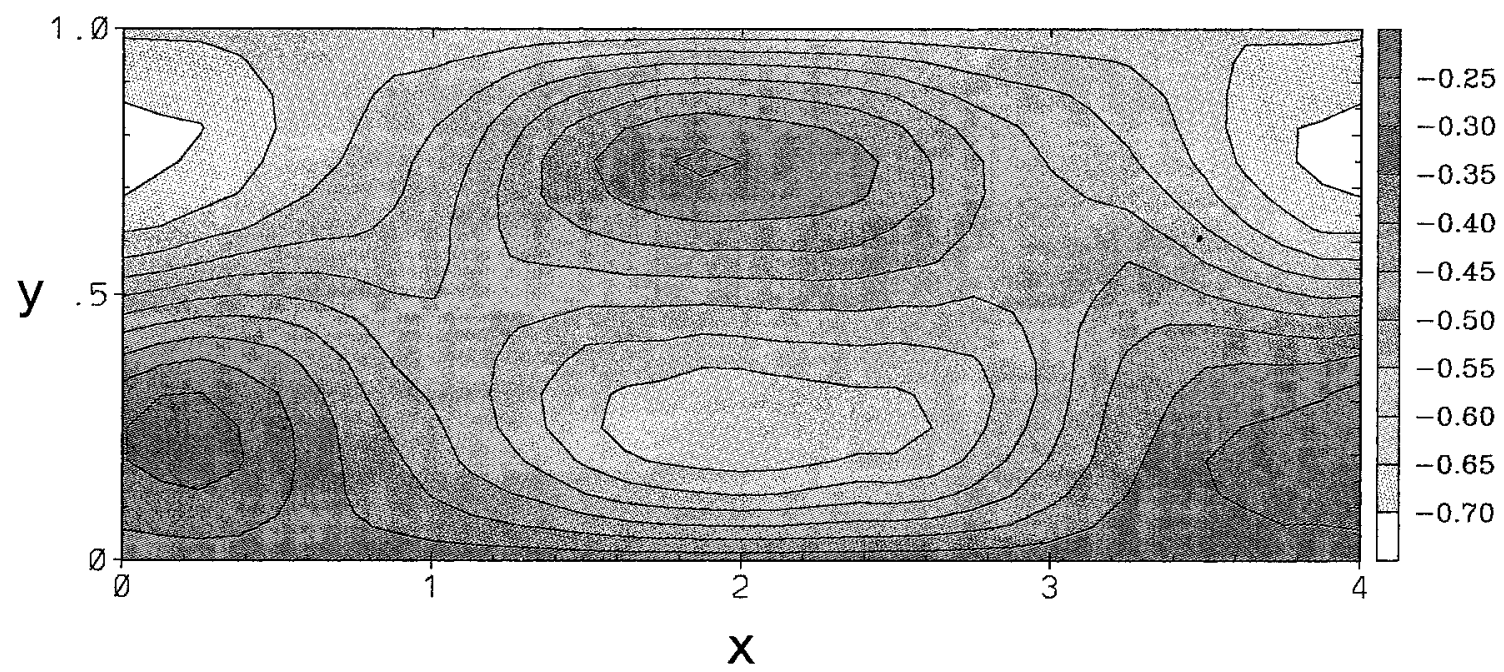

Fig. 11. Snapshot of the upper level total streamfunction at $t=1200$ when $U_{s}=0.95$ in the traditional asymmetric case.

sipative sink in the upper layer to absorb the wave flux of potential vorticity. The only solution is for this flux to be zero; that is, since there is only one wave, the wave amplitude has to be zero.

\section{Wave scale selection}

The preceding numerical results have revealed how a frictional asymmetry affects wave-wave interactions leading to a reinforcement of the wave scale selection. However, they clearly show that this strong wave scale selection is primarily determined by the wave-mean flow interactions. This holds in particular for baroclinic systems with moderate supercriticality whose linear stability analysis does not display any significant effects due to a viscous asymmetry. We have anticipated this result in the introduction, using the argument that, in the process of the nonlinear equilibration, the total zonal flow becomes weakly supercritical. Therefore results about the effects of an asymmetric friction on weakly unstable linear systems should apply. We examine in this section the validity of this argument and, more precisely, what characteristics of the equilibrated zonal flow make it weakly unstable enough for the effects of a traditional asymmetric friction to be significant, leading to a wave scale selection.

For that purpose, we choose to analyze the mean-flow characteristics from a simulation performed with the wave-wave interactions suppressed and characterized by a moderate supercriticality and low dissipation rate ( $U_{s}=0.95$ and $\left.\bar{r} / 2=0.1\right)$. In this simulation the total mean flow (i.e., the total zonal flow) at equilibrium is quasi neutral. So we have chosen to analyze the meanflow characteristics during the transitory period when the selected $(1,2)$ wave monotonically grows. This transitory period lasts for more than 200 time units. During this period the main features of the total mean flow do not differ much from the ones at equilibrium (Rivière
1995). This total mean flow is equal to the basic flow $\left(U_{s}\right)$ plus the mean flow correction $[\bar{u}(y)]$, which varies latitudinally. The mean flow correction is characterized by three main features as revealed by its amplitude spectrum (Fig. 12): (i) the amplitude of the first spectral component of the baroclinic part, which is 15 times larger than the next larger component; (ii) the presence of a significant barotropic part, again dominated by the first spectral component; and (iii) the higher-order meridional structure of the mean flow correction, which, although small, may influence the stability of the system.

A linear stability analysis of this total mean flow, $U_{s}$ $+\bar{u}(y)$, has been carried out. It is a normal-mode analysis performed from Eqs. (1) and (2) linearized around this total mean flow. The method used is the same as the one described in Cai and Mak (1990a). For this total mean flow the normal mode analysis displays only one unstable mode (Fig. 13) dominated by wave $(1,2)$. Its growth rate is small $(\sigma=0.01)$. This confirms the slow growth revealed by the nonlinear results during the transitory period. Furthermore, this growth rate value is an index of the weak supercriticality of the total mean flow since it is much smaller than the ones found in the linear stability analysis of section 3 for a small supercriticality (see Fig. 2). Note again that, with the basic flow alone $\left(U_{s}=0.95\right)$, the baroclinic system in this asymmetric case is unstable with wave $(3,1)$ as the most unstable one with a large growth rate $(\sigma=1.72)$. So the appearance of the mode dominated by $(1,2)$ as the most unstable wave with a small growth rate is due to the nonzero mean flow correction.

In order to find out what features of the mean flow correction make this mode unstable instead of a mode dominated by $(3,1)$, a sensitivity analysis to the three main features mentioned above has been performed using the same normal-mode analysis but with appropriate 

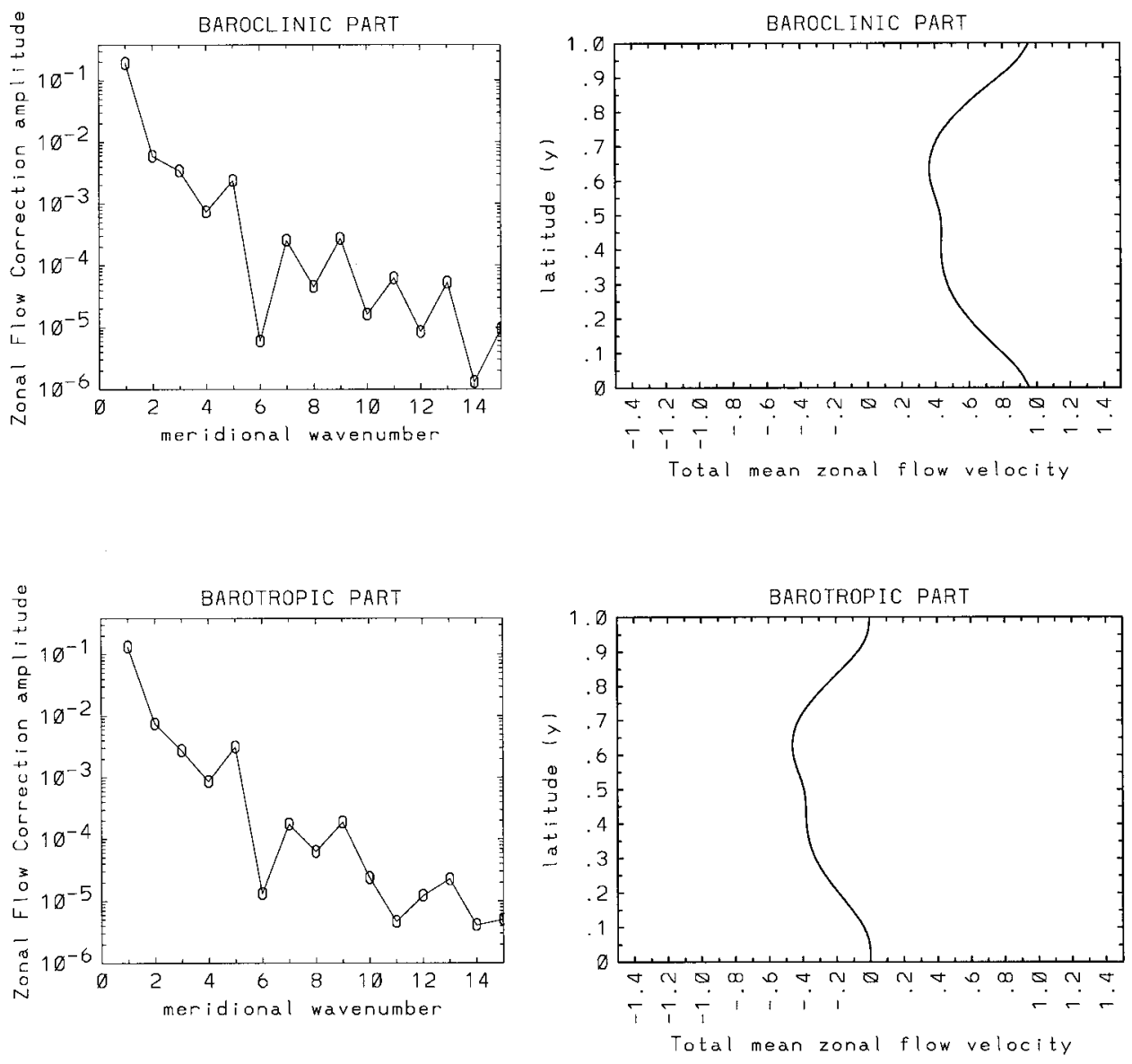

FIG. 12. Amplitude spectrum and $y$ profile of the baroclinic and barotropic part of the total mean zonal current at $t=150$ when $U_{s}=0.95$ in the traditional asymmetric case when the wave-wave interactions are suppressed.

modifications of the mean flow correction. The results are the following.

- When the mean flow correction is truncated to the first spectral component, the normal-mode analysis displays three unstable modes. The first one is the same as the one found above [dominated by $(1,2)$ ] with a growth rate multiplied by 2 . The second one is dominated by $(3,1)$ and the third one by $(2,1)$ with growth rates three times smaller than the first mode. So the higher-order meridional structure, although small, has an effect on the reduction of the instability as indicated by Cai (1992), but its presence does not appear to be important for the wave scale selection.

- When the barotropic zonal jet is suppressed, there is only one very weakly unstable mode dominated by $(2,2)$ with a very small growth rate $(\sigma<0.001)$ : the system is almost neutral. Thus the barotropic zonal jet has a destabilizing role. This can be understood using Pedlosky's (1964) argument: the amplitude and sign (westward) of this barotropic jet is such that its curvature $-\partial^{2} \bar{u}_{B} / \partial y^{2}$ acts to equally reduce the potential vorticity gradient of the total mean flow in both layers (i.e., like having a smaller $\beta$ effect) and therefore to destabilize the otherwise neutral flow. Thus, the presence of the barotropic jet causes the flow to become weakly baroclinically unstable. We have checked that the magnitude of this jet is such that the absolute vorticity in the upper layer does not have any extremum, which makes barotropic instability unlikely.

- When the amplitude of the mean flow correction is reduced by a factor of 1.8 , which leads to increase the total mean shear such that the supercriticality becomes larger, there are two unstable modes. The first is dominated by $(3,1)$ and its growth rate is equal to 0.6 , while the second is dominated by $(1,2)$ with a growth rate equal to 0.1 .

These results show that both the amplitude of the barotropic jet and that of the baroclinic part of the mean flow correction have a significant role on the wave scale selection. The amplitude of the baroclinic part of the mean flow correction is such that the total baroclinic mean flow, when it is considered alone, is almost neutral. However, when the barotropic part is nonzero, the 

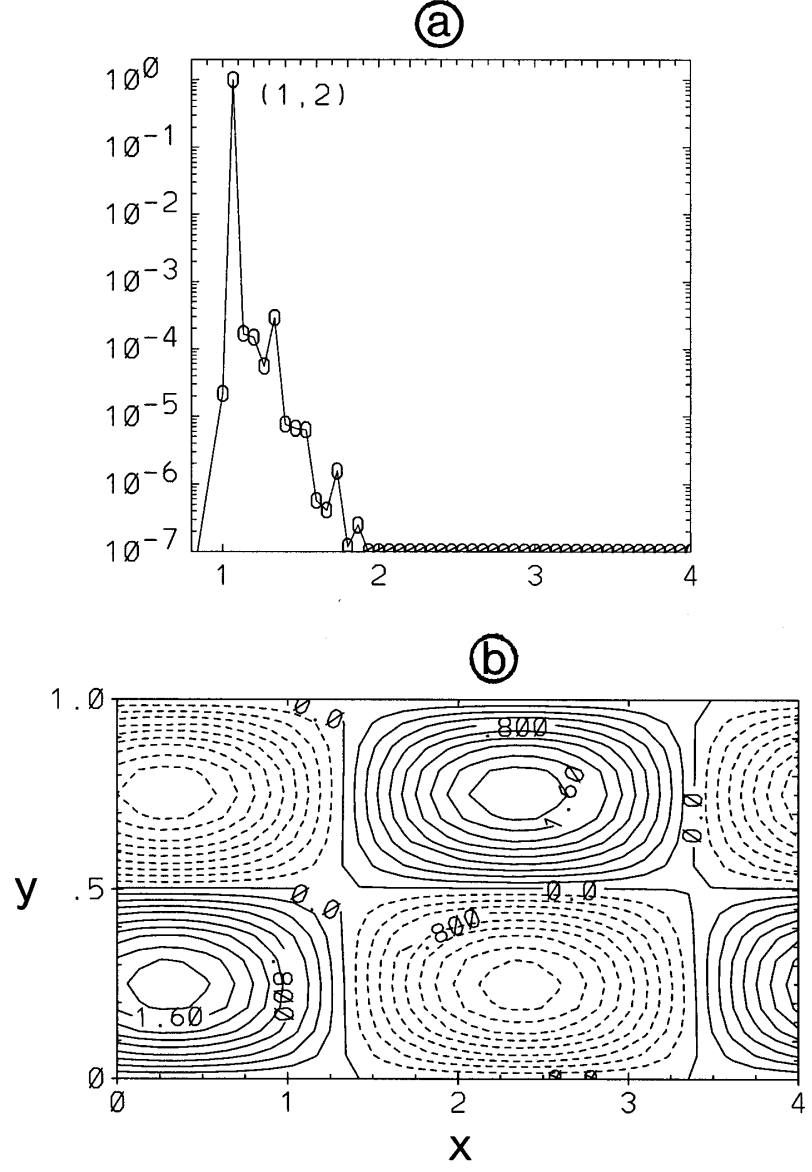

FIG. 13. Upper layer streamfunction (b) and amplitude spectrum (a) of the unstable mode of the total mean flow shown in Fig. 12.

total mean flow is weakly unstable and the most unstable mode is dominated by wave $(1,2)$. It is precisely for this range of supercriticality values that the previous linear studies display the strong effects of an asymmetric friction on the wave scale selection. However, these results show that the weak supercriticality of the total mean flow strongly depends on detailed features of the mean flow correction.

\section{Conclusions}

This study has reexamined the effects of an asymmetric friction on the nonlinear equilibration of baroclinic systems with a particular focus on the wave scale selection. The region of the parameter space explored mostly concerns moderately unstable systems for which a linear stability analysis does not reveal any significant effect of a frictional asymmetry on the most unstable waves. The numerical results obtained do show that the presence and the sense of an asymmetric friction has a strong impact on the nonlinear wave scale selection.

The scenario that emerges is the following: This wave scale selection is primarily induced by wave-mean flow interactions. However, wave-wave interactions increase the differences between the three frictional cases and, in particular, reinforce the wave scale selection. More precisely, in the symmetric case the wave-wave interactions, acting as a process for upscale energy transfer, enlarge the spectral band of the dominant waves, making the long waves emerge. In the nontraditional asymmetric case they are inhibited such that only the synoptic wave selected by the wave-mean flow interactions emerges. Lastly, in the traditional asymmetric case they reduce the spectral wave band and reinforce the emergence of the long wave selected by the wave-mean flow interactions.

The wave scale selection induced by the wave-mean flow interactions when the dissipation is asymmetric can be interpreted as follows. In the process of nonlinear equilibration, the wave-mean flow interactions cause the total mean zonal flow to become weakly supercritical. It is for this range of supercriticality that linear studies reveal the strong effects of an asymmetric friction on the wave scale selection. More precisely, they show that the minimum critical shear is significantly reduced in the traditional asymmetric case compared with other frictional cases (see Fig. 1). Consequently, in the traditional asymmetric case the weakly supercritical nonlinear flow is located in a region of the parameter space that is not accessible by other frictional cases and where the characteristics of the most linearly unstable waves differ. However, the detailed analysis performed in section 6 clearly shows that the weak supercriticality of the nonlinear flow strongly depends on detailed features of the mean flow correction. Consequently the information from the classic linear stability analysis using a uniform baroclinic shear should be considered only as qualitative for the wave scale selection. The amplification of the wave scale selection by the wave-wave interactions, which has been confirmed by an energy budget analysis (Rivière 1995), has been found to be quite robust in the parameter space explored in this study. However, so far we have no interpretation about these effects of an asymmetric friction on the wave-wave interactions. This should be the topic of future study.

A sensitivity analysis has revealed that a large degree of asymmetry is required for an asymmetric friction to significantly affect the nonlinear equilibration of the baroclinic systems. When the degree of the asymmetry is large, the competition between the destabilizing effect of friction and the nonlinear interactions destabilizes the time evolution of the baroclinic waves with a nontraditional asymmetric friction, leading to periodic and chaotic behaviors. No such destabilization is observed with a traditional asymmetric friction or with a symmetric friction except for large supercriticality or when the degree of asymmetry is quite large. This confirms and extends results obtained by previous studies (Chou and Loesch 1986b; Mak 1987). Furthermore, the wave-free equilibrium found when one layer is free of dissipation extends for moderately unstable systems in the $\beta$ plane 
the results of Pedlosky (1983) and Chou and Loesch (1986a). Last, we have found that the higher order stable modes and, in particular, the meridional harmonics of the unstable waves have a large impact on the efficiency of nonlinear interactions whatever the value of the degree of asymmetry is.

In this study we have used the classic Phillips model as a heuristic tool and considered moderately unstable systems for elucidating the effects of frictional asymmetry. Therefore the robustness of these results needs to be investigated for more unstable systems using more realistic models. Several main features have to be questioned when more realistic baroclinic systems are considered. First, does the wave scale selection induced by the wave-mean flow interactions still persist? Indeed, at the equilibrium, the supercriticality of the total mean zonal flow for more unstable systems, although small, is not always so weak as is found in this study. Furthermore, when a basic flow with a nonzero meridional shear is used, the Reynolds stress induced by the wavewave interactions should play a much more important role than it is found in this study. A second feature concerns the effects of an asymmetric friction on the energy transfer between the waves through the wavewave interactions, that is, on the upscale cascade process. Are these effects amplified or reduced when the system is more unstable and the basic flow more realistic and what are the consequences on the inverse energy cascade and the resulting wave scale selection? These questions should be the topic of future study.

Such investigation is stimulated by the aforementioned results of some realistic studies that are reminiscent of ours. Indeed, Cai and Mak (1990b), using a symmetric dissipation, conclude that the wave-wave interactions are the main mechanism that maintains the dominant low-frequency atmospheric waves. On the other hand, Cehelsky and Tung (1991) and Whitaker and Barcilon (1995), who used a traditional asymmetric dissipation, found that the low-frequency atmospheric variability is mostly maintained by the wave-mean flow interactions. However, these studies used numerical models with different physics and different parameter settings. So some work is needed to distinguish and understand the contribution of the effects of the frictional asymmetry in these results.

Acknowledgments. We want to express our thanks to the reviewers for their valuable and stimulating comments. This work is supported by the CNRS and IFREMER. Computations were made on the Cray C98 of the Institut du Développement et des Ressources en Informatique Scientifique (Orsay, France).

\section{REFERENCES}

Cai, M., 1992: An analytic study of the baroclinic adjustment in a quasigeostrophic two-layer channel model. J. Atmos. Sci., 49, 1594-1605.

— , and M. Mak, 1990a: On the basic dynamics of regional cyclogenesis. J. Atmos. Sci., 47, 1417-1442.

- , and — 1990b: Symbiotic relation between planetary and synoptic-scale waves. J. Atmos. Sci., 47, 2953-2968.

Cehelsky, P., and K. K. Tung, 1991: Nonlinear baroclinic adjustment. J. Atmos. Sci., 48, 1930-1947.

Chou, S.-H., and A. Z. Loesch, 1986a: Supercritical dynamics of baroclinic disturbances in a free-surface model. J. Atmos. Sci., 43, 285-301.

$\longrightarrow$, and $-1986 \mathrm{~b}$ : Supercritical dynamics of baroclinic disturbances in the presence of asymmetric Ekman dissipation. $J$. Atmos. Sci., 43, 1781-1795.

Holopainen, E. O., 1961: On the effect of friction in baroclinic waves. Tellus, 13, 363-367.

Hua, B. L., and D. B. Haidvogel, 1986: Numerical simulations of the vertical structure of quasi-geostrophic turbulence. J. Atmos. Sci., 43, 2923-2936.

Klein, P., 1990: Transition to chaos in unstable baroclinic systems: A review. Fluid Dyn. Res., 5, 235-254.

- , and J. Pedlosky, 1986: A numerical study of baroclinic instability at large supercriticality. J. Atmos. Sci., 43, 1243-1262.

Mak, M., 1985: Equilibration in nonlinear baroclinic instability. $J$. Atmos. Sci., 42, 2764-2782.

_ 1987: Dissipative structure of a nonlinear baroclinic system: Effect of asymmetric friction. J. Atmos. Sci., 44, 2613-2627.

McFarlane, N. A., 1987: The effect of orographically excited gravity wave drag on the general circulation of the lower stratosphere and troposphere. J. Atmos. Sci., 44, 1775-1800.

Palmer, T. N., G. J. Shutts, and R. Swinbank, 1986: Alleviation of a systematic westerly bias in general circulation and numerical weather prediction models through an orographic gravity wave drag parameterization. Quart. J. Roy. Meteor. Soc., 112, 10011040 .

Pedlosky, J., 1964: The stability of currents in the atmosphere and the ocean: Part II. J. Atmos. Sci., 21, 342-353.

- 1981: The effect of $\beta$ on the chaotic behavior of unstable baroclinic waves. J. Atmos. Sci., 38, 717-731.

, 1983: The growth and decay of finite-amplitude baroclinic waves. J. Atmos. Sci., 40, 1863-1876.

- 1987: Geophysical Fluid Dynamics. 2d ed. Springer-Verlag, $710 \mathrm{pp}$.

Phillips, N. A., 1954: Energy transformations and meridional circulations associated with simple baroclinic waves in a two-level quasi-geostrophic model. Tellus, 6, 273-286.

Rivière, P., 1995: Effets de la friction sur l'instabilité barocline non linéaire. Ph.D. thesis, l'Université de Bretagne Occidentale, 169 pp. [Available from the Université de Bretagne Occidentale, 29280 Brest, France.]

Romea, R. D., 1977: The effect of friction and beta on finite amplitude baroclinic waves. J. Atmos. Sci., 34, 1689-1695.

Wang, B., 1990: On the asymmetry of baroclinic instability between easterly and westerly shear. Tellus, 42A, 463-468.

Weng, H.-Y., and A. Barcilon, 1991: Asymmetric Ekman dissipation, sloping boundaries and linear baroclinic instability. Geophys. Astrophys. Fluid Dyn., 59, 1-24.

Whitaker, J. S., and A. Barcilon, 1995: Low frequency variability and wavenumber selection in models with zonally symmetric forcing. J. Atmos. Sci., 52, 491-503. 